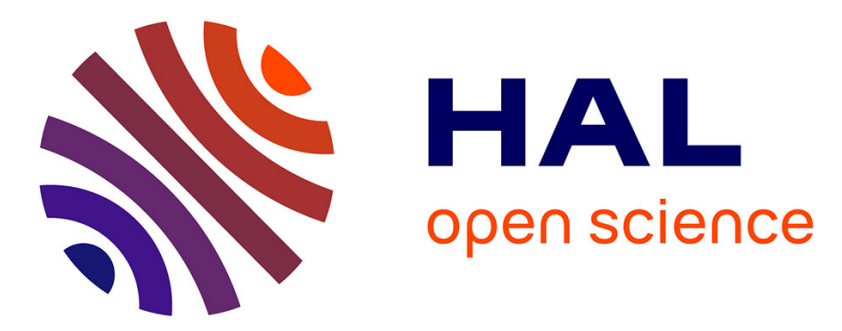

\title{
Personnaliser des activités pédagogiques de manière unifiée: une solution à la diversité des dispositifs
}

Marie Lefevre, Nathalie Guin, Stéphanie Jean-Daubias

\section{To cite this version:}

Marie Lefevre, Nathalie Guin, Stéphanie Jean-Daubias. Personnaliser des activités pédagogiques de manière unifiée: une solution à la diversité des dispositifs. STICEF (Sciences et Technologies de l'Information et de la Communication pour l'Éducation et la Formation), 2012, 19, pp.309-351. hal01353061

\section{HAL Id: hal-01353061 \\ https://hal.science/hal-01353061}

Submitted on 11 Jun 2021

HAL is a multi-disciplinary open access archive for the deposit and dissemination of scientific research documents, whether they are published or not. The documents may come from teaching and research institutions in France or abroad, or from public or private research centers.
L'archive ouverte pluridisciplinaire HAL, est destinée au dépôt et à la diffusion de documents scientifiques de niveau recherche, publiés ou non, émanant des établissements d'enseignement et de recherche français ou étrangers, des laboratoires publics ou privés. 


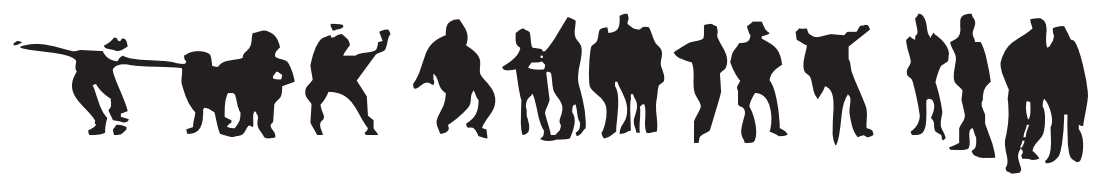

\section{Personnaliser des activités pédagogiques de ma- nière unifiée : une solution à la diversité des dispositifs}

Marie LEFEVRE, Nathalie GUIN, Stéphanie JEAN-DAUBIAS (LIRIS, Lyon)

RÉSUMÉ • Dans cet article, nous abordons la question de la personnalisation des activités d'apprentissage en cherchant une solution au problème causé par la diversité des dispositifs utilisables par les enseignants dans leur préparation des activités des apprenants. Pour cela, nous adoptons une approche générique qui s'appuie sur PERSUA2, un modèle pour la personnalisation unifiée des activités d'apprentissage. Dans cet article, nous nous appuyons sur des scénarios d'usage pour décrire les besoins en matière d'individualisation de l'enseignement. Nous présentons ensuite le modèle PERSUA2 et la mise en œuvre que nous en avons faite, qui permet à un enseignant d'expliciter une stratégie de personnalisation qui est ensuite appliquée par le système établissant des activités personnalisées pour les élèves. Nous terminons par les évaluations que nous avons menées, qui montrent que notre approche permet de répondre aux différents besoins identifiés par les scénarios d'usage.

MOTS CLÉS - Personnalisation des activités d'apprentissage, approche unifiée, stratégies pédagogiques, modèle.

ABSTRACT - In this paper, we address the issue of personalization of learning activities by seeking a solution to the problem caused by the variety of devices used by teachers in preparing activities for learners. For this, we adopt a generic approach based on PERSUA2, a model for a unified personalization of learning activities. In this paper, we rely on use cases to describe needs for individualization of teaching. We then present the PERSUA2 model and, the implementation that we have made, which enable a teacher to define a personalization strategy, which is then applied by the system that prepares customized activities for students. We conclude by reporting evaluations that we conducted, which show that our approach can satisfy the different needs identified by the use cases.

KEYWORDS • Personalization of learning, unified approach, teaching strategies, model.

Marie LEFEVRE, Nathalie GUIN, Stéphanie JEAN-DAUBIAS, Personnaliser des activités pédagogiques de manière unifiée : une solution à la diversité des dispositifs, Revue STICEF, en ligne sur www.sticef.org 


\section{Introduction}

La personnalisation des activités d'apprentissage est une problématique fondamentale dans les recherches sur les Environnements Informatiques pour l'Apprentissage Humain (EIAH) (Marty et Mille, 2009). Elle permet en effet d'une part d'améliorer l'apprentissage en proposant des contenus plus pertinents prenant en compte les connaissances et compétences des apprenants (éventuellement en adaptant la manière et la temporalité avec lesquelles ont propose ces contenus) (Wenger, 1987) (Brusilovsky, 2001), et d'autre part de proposer des contenus plus accessibles, en prenant en compte les capacités des utilisateurs et leurs éventuels handicaps. Si elle est abordée de plus en plus fréquemment dans les travaux de recherche, si de plus en plus d'EIAH offrent ou cherchent à offrir des possibilités de personnalisation à leurs utilisateurs apprenants et/ou enseignants (Lefevre et al., 2011b), la situation des enseignants souhaitant personnaliser l'apprentissage de leurs élèves n'est pas entièrement satisfaisante pour autant. En effet, s'ils ont maintenant à disposition quelques EIAH personnalisables qu'ils peuvent adapter parfois à leurs habitudes, parfois aux besoins de leurs élèves, ces différents outils sont hétérogènes: ils s'utilisent différemment, ne proposent pas tous les mêmes fonctionnalités, et diffèrent des procédures employées pour personnaliser des activités papier-crayon. La tâche de personnalisation est donc complexe et coûteuse en temps pour l'enseignant.

L'approche que nous proposons vise à proposer un cadre unificateur permettant de guider l'enseignant dans la personnalisation des activités pédagogiques. Elle lui permet de s'appuyer sur les connaissances des apprenants, fournies par des profils issus de sources diverses, pour définir des séances de travail personnalisées contenant des activités tant sous forme papier (des feuilles exercices qui seront générées de façon adaptée aux besoins exprimés) que sous forme logicielle (des sessions également entièrement personnalisées au sein d'EIAH existants). Cette définition de séances de travail personnalisées variées se fait au sein d'un unique dispositif, exploitant les informations sur les apprenants, et servant d'interface entre l'enseignant et les différents EIAH à personnaliser. Cette approche permet de prendre en compte différents niveaux et les disciplines les plus variées.

Pour mettre en place une telle approche de personnalisation, il est nécessaire de prendre en compte les spécificités des apprenants, en s'appuyant par exemple sur leur profil d'apprentissage, mais il est également pertinent de prendre en compte les besoins des enseignants, que nous qualifions de stratégie pédagogique. Nous définissons un profil d'apprentissage comme un ensemble d'informations, concernant 2 
l'apprentissage d'un apprenant ou d'un groupe d'apprenants, collectées ou déduites à l'issue d'une ou plusieurs activités pédagogiques, qu'elles soient ou non informatisées. Les informations contenues dans le profil d'apprentissage d'un apprenant peuvent concerner ses connaissances, compétences, conceptions, son comportement, ou encore des informations d'ordre métacognitif (Jean-Daubias, 2011). Ces informations peuvent détailler très finement les spécificités des apprenants, elles sont donc une source riche pour la personnalisation des activités qui leur seront proposées.

Pour fournir un dispositif de personnalisation tel que nous le décrivons, nous nous appuyons sur PERSUA2, notre modèle de PERsonnalisation Unifiée des Activités d'Apprentissage, sur lequel nous centrons cet article. Ce modèle associe stratégie pédagogique et contexte d'utilisation. La stratégie pédagogique de l'enseignant définit comment affecter des activités aux apprenants. Cette affectation est décrite à l'aide d'un ensemble de liens hiérarchisés qui associent des contraintes sur le profil des apprenants à des contraintes sur une ou plusieurs activités pédagogiques. Les contraintes sur le profil permettent de sélectionner un ensemble d'apprenants ayant une ou plusieurs caractéristiques donnée(s) dans leur profil. Les contraintes sur les activités permettent d'adapter les activités (exercices papier à imprimer ou activités sur un EIAH) en fonction des caractéristiques sélectionnées au niveau des profils. Le contexte d'utilisation décrit l'ensemble des informations permettant de caractériser la situation d'apprentissage au moment de la séance de travail (apprenants concernés, nombre d'exercices, durée, logiciels disponibles...).

Dans la section 2 de cet article, nous présentons trois scénarios d'usage illustrant les verrous liés à la personnalisation de l'enseignement. Dans la section 3, nous présentons les forces et les faiblesses des approches proposées actuellement pour aider les enseignants dans leur tâche de personnalisation. Dans la section 4, nous décrivons l'approche que nous avons retenue afin de proposer un outil d'aide à la personnalisation. Dans la section 5, nous présentons le modèle PERSUA2 sur lequel s'appuie notre outil. Dans la section 6, nous présentons la mise en œuvre de ce modèle et les évaluations que nous avons menées, avant de revenir sur les scénarios d'usage initiaux pour montrer en quoi nos propositions changent ces situations. Dans la section 7, nous discutons nos propositions avant de conclure cet article en présentant les perspectives soulevées par ce travail. 


\section{Scénarios d'usage}

Nous présentons dans cette section trois scénarios montrant les enjeux de la personnalisation des activités pédagogiques dans les pratiques des enseignants. Nous montrons ensuite les limites freinant leur mise en œuvre, ainsi que les questions de recherche associées. Par la suite, nous reviendrons sur le premier scénario pour illustrer l'utilisation du modèle PERSUA2 proposé en réponse à ces questions, puis nous reprendrons l'ensemble des scénarios lors de l'évaluation de nos propositions pour montrer en quoi ces dernières peuvent changer les situations décrites.

\subsection{Aide personnalisée à l'école primaire}

Dimitri, enseignant de CE2 de l'enseignement français, imprime en début d'année les bilans personnels de ses élèves de 8 ans proposés par le logiciel J'ADE (J'ADE, 2007). Ces bilans correspondent aux résultats des évaluations nationales en français et en mathématiques que ses élèves ont passées à la fin de l'année précédente, à la demande de l'institution scolaire. Il fait également régulièrement utiliser à ses élèves le logiciel AMBRE-add (Guin et al., 2011) pour l'apprentissage de la résolution de problèmes additifs, logiciel qu'ils utilisent en autonomie lorsqu'ils ont terminé leur travail.

Dans l'établissement où enseigne Dimitri, une aide personnalisée est proposée aux élèves en difficulté. Pour cela, chaque enseignant identifie les élèves concernés dans sa classe. Ensuite les enseignants forment des groupes de six élèves. Chaque élève bénéficiera d'une heure de soutien en plus des heures de cours, dispensée par un autre enseignant que le sien. Pour préparer le contenu de ces séances de soutien, les enseignants se rencontrent une heure par semaine : chaque enseignant fixe les objectifs à atteindre par ses élèves et fournit à ses collègues la liste des exercices à faire, tirés de manuels scolaires.

Dimitri souhaiterait s'appuyer sur toutes les informations dont il dispose sur ses élèves (données issues des évaluations nationales, profils d'élèves créés par le logiciel AMBRE-add, résultats en classe, bilan d'orthophoniste, observations des enseignants relatives par exemple à un problème de concentration, etc.) pour proposer des séances de remédiation, soit sous forme papier, soit avec des logiciels d'apprentissage, adaptées à chacun des élèves concernés par les séances de soutien. 


\subsection{Combiner remédiation et approfondissement}

Hélène, professeur d'anglais au collège, ne fait actuellement ni remédiation, ni approfondissement avec ses classes de trente élèves.

Elle souhaiterait, avec chacune de ses classes, faire une séance d'une heure en salle informatique durant laquelle elle séparerait la classe en deux groupes : les élèves ayant des difficultés dans le premier et les autres dans le second. La salle informatique de son établissement contient d'un côté des ordinateurs et de l'autre des tables et un tableau. Pendant la première demi-heure de chaque séance, le groupe d'élèves sans difficulté apprendrait du nouveau vocabulaire grâce aux logiciels Planet English (Planet English, 2006) et VocabOne (VocabOne, 2007). De son côté, elle ferait travailler le groupe d'élèves en difficulté sur des points non compris précédemment grâce à des discussions orales et à des exercices papier qu'elle inventerait. Durant la seconde demi-heure, Hélène échangerait les groupes. Les élèves en difficulté passeraient sur ordinateur pour faire des activités adaptées à leurs difficultés de prononciation sur le logiciel Sephonics (Sephonics, 2008), tandis qu'elle travaillerait avec les autres élèves pour approfondir des notions vues en cours.

Durant cette séance, elle souhaiterait que lors de l'utilisation de l'un des trois logiciels, chaque élève se voie proposer un contenu adapté à ses besoins, en tenant compte de ses connaissances et de ses lacunes.

\subsection{Recommandation de ressources pédagogiques à l'université}

Lucie est maître de conférences en informatique. Dans le cadre de ses enseignements, elle utilise un Espace Numérique de Travail. Dans cet ENT, elle dispose d'un profil pour chacun de ses étudiants, profil composé de différentes notes, de commentaires et d'un ensemble de compétences à acquérir. L'ENT lui permet de mettre à disposition des étudiants des ressources pédagogiques (cours ou exercices de types divers).

Actuellement, elle propose ces ressources, tout au long de l'année, à l'ensemble des étudiants, mais elle souhaiterait pouvoir proposer progressivement les ressources aux étudiants en fonction des connaissances qu'ils ont acquises, représentées dans leur profil. 


\subsection{Les besoins en termes de personnalisation}

Avec les outils actuellement à la disposition des enseignants, le travail qu'ils doivent effectuer pour mettre en œuvre ces scénarios est très lourd.

En effet, pour chaque élève qu'il souhaite envoyer en soutien, Dimitri doit analyser d'une part ses résultats aux évaluations nationales, d'autre part son profil créé par le logiciel AMBRE-add, et enfin les résultats de l'élève en classe ainsi que les sources d'informations extérieures (bilan d'orthophoniste par exemple). À partir de l'ensemble de ces informations, il doit identifier les difficultés de l'élève et choisir dans des manuels scolaires des exercices adaptés à ces difficultés, exercices qu'il photocopie et organise pour constituer une fiche d'exercices.

En ce qui concerne Hélène, elle souhaite utiliser trois logiciels existants pour l'apprentissage de l'anglais, avec un contenu adapté aux compétences et difficultés de chacun de ses élèves. Elle doit donc apprendre à paramétrer le logiciel VocabOne et étudier le contenu des logiciel Sephonics et Planet English pour connaître les exercices qu'ils contiennent. Ensuite, pour chacun de ses 30 élèves, elle doit définir une séquence d'exercices en fonction de ce qu'elle connaît de l'élève, et soit paramétrer le logiciel VocabOne pour qu'il propose les exercices souhaités, soit donner à chaque élève la liste des exercices à faire sur les logiciels Sephonics et Planet English.

Quant à Lucie, elle dispose d'un profil pour chacun des étudiants qui utilisent l'ENT, mais elle doit choisir manuellement pour chacun d'entre eux des ressources pédagogiques existantes dans la plateforme afin de leur constituer des séquences de travail personnalisées.

Ces trois scénarios montrent les difficultés que rencontrent les enseignants souhaitant mettre en place une personnalisation de l'enseignement. Ainsi, si un enseignant souhaite adapter à ses habitudes de travail les activités pédagogiques proposées aux apprenants, il doit maîtriser une multitude d'outils. Il a le choix entre créer ses exercices sur papier, utiliser différents générateurs d'exercices, modifier les fichiers de configuration des EIAH ou se servir des interfaces de paramétrage des EIAH lorsqu'elles existent. Pour les outils informatisés, il doit à chaque fois prendre en main un nouvel environnement, puisque d'un outil à l'autre, les interfaces et les principes d'utilisation diffèrent. De plus, s'il souhaite adapter les activités aux informations dont il dispose sur chaque apprenant (ses observations, divers profils papiers ou informatisés), il doit traiter manuellement l'ensemble des informations pour chaque apprenant. Cette complexité est illustrée sur la Figure 1. 


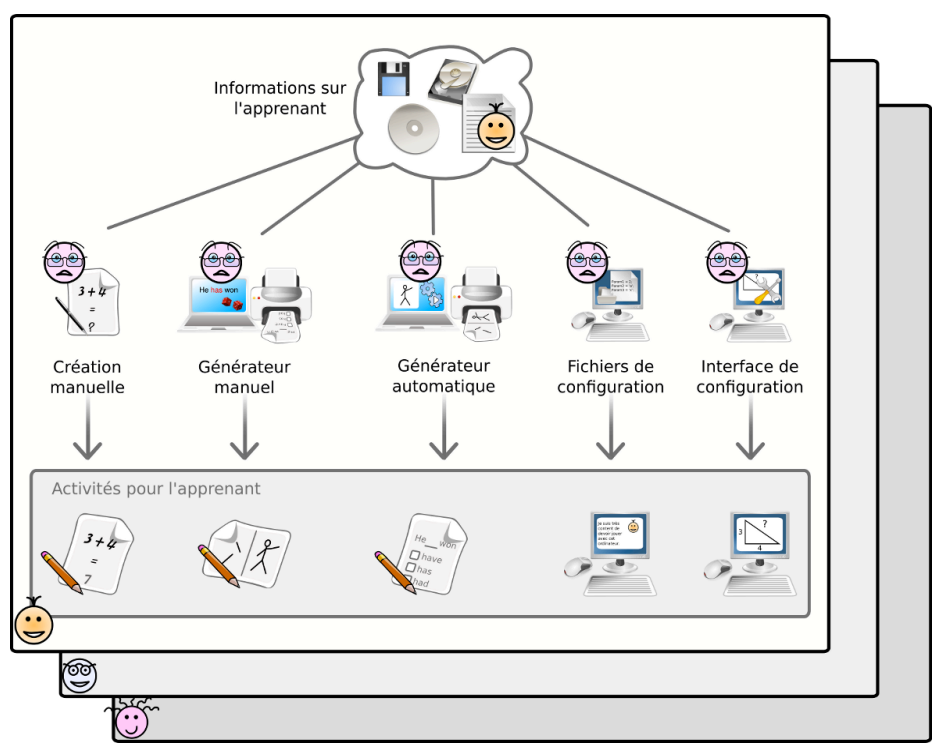

Figure 1 • Principe de la personnalisation par un enseignant des activités pédagogiques pour chacun de ses élèves

Pour dépasser ces limites et permettre la réalisation des différents scénarios présentés, il est nécessaire de s'intéresser aux verrous suivants :

- Comment prendre en compte les individualités des apprenants? Et comment diminuer la charge de travail d'un enseignant quand il traite les informations relatives aux apprenants ?

- Comment prendre en compte les besoins et habitudes de travail des enseignants concernant les choix des activités à utiliser dans leur enseignement ? Et donc comment homogénéiser les outils de personnalisation des activités pédagogiques (générateurs d'exercices papier et interfaces de paramétrage des EIAH) du point de vue de l'enseignant?

- Comment aider l'enseignant à affecter aux apprenants des activités correspondant à ses habitudes de travail en fonction des individualités de chaque apprenant ?

\section{Approches relatives à la personnalisation de l'apprentissage}

Nous venons de voir que les verrous liés à la mise en œuvre d'une personnalisation des activités d'apprentissage par les enseignants sont variés. Ainsi, les approches proposées dans le cadre de la personnalisation de l'apprentissage le sont aussi : elles 
traitent de questions de recherche précises, mais différentes. Certaines s'intéressent à l'adaptation à l'apprenant, d'autres à l'adaptation à l'enseignant, d'autres encore à la façon d'affecter les activités aux apprenants. L'adoption exclusive de l'une de ces approches n'est pas entièrement satisfaisante. En effet, adapter l'enseignement uniquement selon les besoins de l'enseignant permet à ce dernier de préparer une session conforme à ses souhaits, mais exclut la personnalisation en fonction des spécificités de chaque apprenant. Certains travaux, dans lesquels nous nous reconnaissons, font le choix de combiner plusieurs de ces approches. Pour pouvoir proposer une personnalisation des activités d'apprentissage, qui soit efficace, acceptée et utilisée par les enseignants dans leurs pratiques, nous considérons en effet qu'il est nécessaire de proposer des outils qui tiennent compte de ces trois axes en même temps. C'est pourquoi nous proposons de discuter des forces et des limites des approches existantes en considérant les trois critères d'analyse suivants : la prise en compte des individualités des apprenants, la prise en compte des besoins et habitudes pédagogiques des enseignants et l'affectation d'une activité à un apprenant.

\subsection{Prendre en compte les individualités des apprenants}

La prise en compte des individualités des apprenants nécessite de disposer d'informations sur ces derniers (leurs connaissances, leur parcours d'apprentissage, les erreurs qu'ils font, leurs éventuels handicaps), provenant de l'apprenant lui-même, des traces de ses activités d'apprentissage antérieures, des indications données par l'enseignant, Ces informations sont classiquement stockées dans des profils d'apprenant (Mitrovic, 1998), (Sørmo et al., 2002). Un profil d'apprenant est spécifique à un individu. Il encode finement les informations déduites sur les apprenants suites aux différentes activités pédagogiques qu'ils ont réalisées. Toutefois, le processus de construction des profils d'apprenant n'est pas sans risque et des erreurs de diagnostic peuvent conduire à la prise en compte d'informations erronées.

L'utilisation de stéréotypes permet à la fois de prendre en compte cette limite et de simplifier le processus d'intégration des informations sur l'apprenant et le processus de personnalisation. Un stéréotype contient un ensemble de caractéristiques que l'on trouve chez plusieurs apprenants (Rich, 1983). Le système dispose de plusieurs stéréotypes et chaque apprenant doit être associé à l'un de ces stéréotypes. Cette factorisation a le défaut de ne pas permettre de prise en compte fine des individualités des apprenants, puisque deux apprenants aux connaissances différentes peuvent être associés à un même stéréotype. En EIAH, plusieurs travaux ont fait le choix d'adopter cette approche (Vincent et al., 2005), (Girard et al., 2007).

$$
8
$$


Dans les deux approches, toute la difficulté consiste à capturer des données pertinentes concernant les apprenants et à donner les moyens à l'enseignant ou au système mettant en œuvre la personnalisation d'accéder facilement à ces données, afin de les utiliser pour la personnalisation des activités.

\subsection{Prendre en compte les besoins et habitudes pédagogiques des en- seignants}

Chaque enseignant a ses propres habitudes de travail : certains préfèrent par exemple travailler avec des activités sur support papier, d'autres utilisent un ou plusieurs logiciels pédagogiques.

Si nous prenons l'exemple de l'enseignement de l'addition en CE1, certains enseignants préfèrent travailler sur papier, d'autres sur ardoise, d'autres avec des logiciels. Certains préfèrent des séances répétées de 3 minutes, d'autres préfèrent une seule séance de 20 minutes. Certains présenteront les tables dans l'ordre (de 1 à 10), d'autres commenceront par les doubles, d'autres encore commenceront par les tables de 1, 2, 5, 10, puis feront travailler les autres. À cela s'ajoute des contraintes organisationnelles : disponibilités de la salle informatique, nombre d'élèves, horaire des récréations.

Pour un même enseignement, les habitudes et besoins de chaque enseignant peuvent être extrêmement variés. Pour prendre en compte ces spécificités, et leur permettre de personnaliser leur enseignement, une première approche consiste à utiliser des outils auteurs. De nombreux outils auteurs ont été proposés, permettant de définir des ressources pédagogiques variées. Certains permettent de créer des exercices (David et al., 1996), d'autres des logiciels pédagogiques (Van Joolingen et al., 2003), d'autres enfin des hypermédias adaptatifs (Cristea et Carro, 2008). Pour cela, l'enseignant doit fournir entièrement le contenu pédagogique de la ressource. Certains outils auteurs tels que REDEEM (Ainsworth, 2000), GTE (Van Marcke, 1998), Smart Trainer (Chen et al., 1998) et Eon (Murray, 2003a) offrent de plus aux enseignants créant un logiciel pédagogique la possibilité de paramétrer des modèles de stratégies pédagogiques. Ces stratégies pédagogiques sont ensuite mises en œuvre lors de l'utilisation par l'apprenant du logiciel créé. Ce principe est également proposé par des outils auteurs permettant de créer des hypermédias adaptatifs (Cristea, 2005). Quel que soit le type de ressources créé avec les outils auteurs, leur utilisation permet aux enseignants d'obtenir des ressources qui leur sont adaptées, mais cette utilisation est coûteuse en temps. 
Une seconde approche consiste à utiliser des scénarios pédagogiques qui définissent les activités proposées aux apprenants, en précisant le contexte dans lequel ils se trouvent, le rôle de chaque participant et les actions à accomplir sur les ressources (Pernin et al., 2004). Les outils de définition de scénarios ne permettent cependant pas de créer des ressources, mais seulement d'associer des ressources existantes, qui sont soit contenues dans l'application sur laquelle le scénario sera mis en œuvre, soit créées manuellement par l'enseignant, soit créées en utilisant une application spécifique (iClass, 2004).

Une autre approche consiste à réserver une partie du logiciel pédagogique aux enseignants ou à leur proposer un module spécifique leur permettant de paramétrer l'environnement proposé à l'apprenant. Ces espaces réservés aux enseignants permettent pour certains d'adapter le contenu pédagogique (Duclosson et al., 2005) et/ou l'interface (Leroux, 2002), pour d'autres de définir des stratégies pédagogiques (Murray, 2003a). Toutefois, peu de logiciels possèdent un tel espace pour l'enseignant et, lorsqu'ils existent, ces espaces diffèrent d'un logiciel à l'autre. Ainsi, un enseignant voulant utiliser plusieurs systèmes devra maîtriser les différents outils de paramétrage correspondants.

En conclusion, pour prendre en compte les besoins et habitudes de chaque enseignant, des solutions variées ont été proposées, mais l'hétérogénéité des différents systèmes constitue en elle-même une limite du point de vue de l'enseignant. En effet, pour adapter des activités de diverses provenances (issues de générateurs, décrites dans des scénarios, contenues dans des logiciels, etc.) à ses buts pédagogiques, l'enseignant doit apprendre à utiliser l'interface de nombreux outils et il n'existe pas d'approche unifiée permettant d'assister l'enseignant dans cette tâche d'adaptation des activités.

\subsection{Permettre l'affectation des activités}

Le choix des activités proposées à l'apprenant peut être fait directement par les EIAH. La personnalisation est alors effectuée en fonction de la connaissance que le système a de l'apprenant. Cette connaissance peut être simple, par exemple des informations sur les réponses de l'apprenant (Burton, 1982), ou plus complexe, par exemple sous forme de modèle de l'apprenant (Mitrovic, 1998). Ces EIAH sont donc personnalisés en fonction de chaque apprenant, mais cette personnalisation n'est pas forcément en adéquation avec les objectifs pédagogiques des enseignants. 
D'autres systèmes permettent à l'apprenant de réguler lui-même son apprentissage en choisissant un thème ou une connaissance à étudier (Melis et al., 2001), ou plus directement une activité à effectuer (VanLehn et al., 2005). Cette «autopersonnalisation » peut être efficace lorsque l'apprenant travaille en autonomie (en complément de l'enseignement classique, en formation à distance...) mais dans le cadre d'un enseignement supervisé (en classe par exemple), il est difficile pour l'enseignant de s'assurer que chaque élève travaille sur des activités conformes aux objectifs définis en classe.

D'autres systèmes enfin permettent à l'enseignant d'intervenir dans les choix d'affectation des activités aux apprenants. Certains systèmes fournissent une interface de paramétrage permettant de choisir les activités que feront les apprenants (Leroux, 2002), (Duclosson et al., 2005) ou de définir des règles d'affectation (Murray, 2003a). Certains systèmes auteurs permettent de définir des stratégies pédagogiques dès la création du logiciel (Murray, 2003b). D'autres systèmes enfin n'offrent pas d'espace pour l'enseignant, mais sont modifiables via des fichiers de configuration. Dans tous les cas, l'enseignant doit bien connaître le logiciel à personnaliser pour réussir à définir les paramètres permettant que le logiciel corresponde à ses besoins. De plus, pour réaliser cette affectation d'activités, l'enseignant n'a pas facilement accès aux informations relatives aux apprenants. Cette personnalisation est donc souvent longue à mettre en place.

D’une manière générale, les choix pédagogiques intervenant dans la personnalisation, et donc dans l'affectation d'une activité à un apprenant, doivent être effectués par l'enseignant si l'on souhaite que la personnalisation mise en œuvre corresponde à ses buts et à ses besoins pédagogiques. Or, rares sont les systèmes qui permettent de prendre en compte ces besoins.

\section{Notre approche de la personnalisation des activités pédagogiques}

L'étude de l'existant montre que de nombreuses solutions ont été proposées pour faciliter la mise en place de la personnalisation de l'enseignement. Ce que nous proposons, c'est un cadre unificateur permettant de guider l'enseignant dans la personnalisation des activités d'apprentissage, ayant pour support un outil unique utilisé par l'enseignant. Cet outil intègre les diverses informations sur les apprenants et permet d'adapter les activités aux élèves et aux besoins pédagogiques de l'enseignant, à travers une interface unique (cf. Figure 2). Pour cela, cet outil s'appuie sur des mo- 
dèles de personnalisation définis en fonction des buts pédagogiques de chaque enseignant.

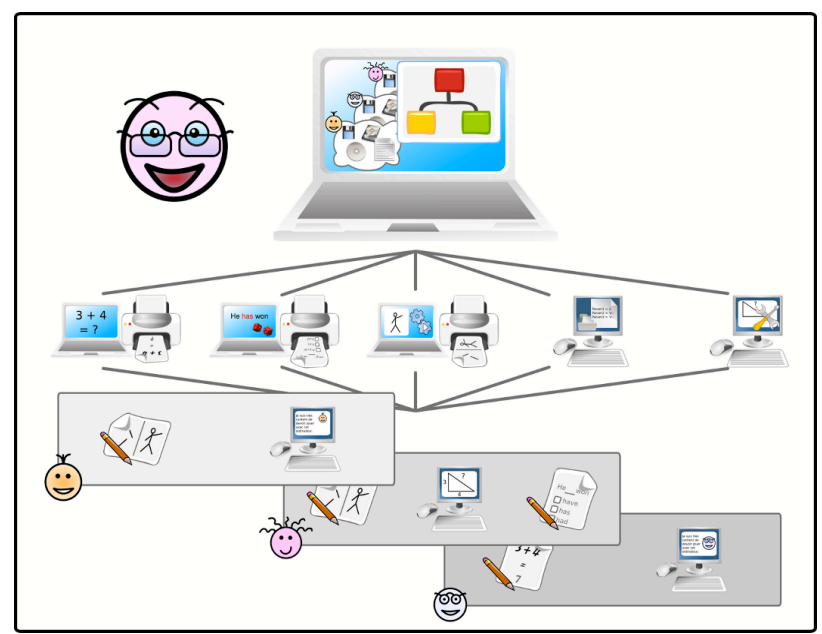

Figure 2 • Principe d'une personnalisation unifiée des activités pédagogiques

Notre approche repose sur la formalisation des différents éléments nécessaires à la personnalisation. Tout d'abord, les informations relatives aux apprenants issues de sources diverses peuvent être décrites dans un même formalisme. Dans notre approche, nous utilisons le langage de description de profils PMDLe (Eyssautier-Bavay, 2008) (Jean-Daubias et Ginon, 2010). Nous avons augmenté ce langage en proposant le modèle cPMDLe (Lefevre, 2009) (Ginon et Jean-Daubias, 2010) permettant de contraindre les éléments d'un profil pour en sélectionner une partie.

De même, la création d'activités, papier ou logicielles, repose sur des modèles implicites ou explicites, que nous formalisons grâce à l'approche GEPPETO (Lefevre, 2010). Cette formalisation permet aux enseignants de contraindre l'adaptation des activités papier ou logicielles pour qu'elles correspondent à leurs habitudes de travail.

Enfin, il est possible de définir des modèles de personnalisation permettant d'acquérir les pratiques éducatives des enseignants. Nous proposons pour cela le modèle PERSUA2, détaillé dans la section suivante. Ce modèle permet de faire le lien entre des éléments du profil de l'apprenant sélectionnés grâce à notre modèle cPMDLe et des activités définies grâce à notre approche GEPPETO. Un processus 
d'exploitation du modèle de personnalisation ainsi défini permettra d'affecter à un apprenant dont le profil respecte certaines caractéristiques, les activités pédagogiques prévues par l'enseignant.

L'utilisation du modèle PERSUA2 permet à chaque enseignant de mener une tâche réflexive sur son enseignement et sur ses stratégies d'individualisation en les formalisant explicitement dans un modèle, notamment sous forme de règles. Ces règles, qui forment la stratégie pédagogique d'un enseignant, peuvent représenter autant la part de routine dans la pratique des enseignants (Perrenoud, 2000), c'est-àdire la part de tradition collective que chaque enseignant reprend à son compte lorsqu'il crée ses enseignements, que le style d'enseignement (Clot et Faïta, 2000), c'està-dire la manière propre à chaque enseignant d'utiliser les ressources pédagogiques dont il dispose, que l'habitus de chaque enseignant (Bourdieu, 1980), si celui-ci arrive à identifier et à formaliser les schèmes de perception et d'actions qu'il met en œuvre inconsciemment lors de son enseignement. Chaque modèle de personnalisation défini selon le modèle PERSUA2 peut alors être partagé, échangé et réutilisé entre enseignants.

5. PERSUA2 : un modèle pour une personnalisation unifiée des activités d'apprentissage

Pour personnaliser une séance de travail, il est nécessaire d'avoir des informations sur l'apprenant (connaissances, compétences, etc.) auquel la séance de travail est destinée, mais également sur la situation dans laquelle cette séance va se dérouler (lieu, durée, but pédagogique, etc.). La combinaison de ces deux types d'informations au sein d'un même dispositif rend possible la création de séances de travail adaptées d'une part à l'apprenant et d'autre part aux buts pédagogiques de l'agent de la personnalisation (l'enseignant ou l'EIAH).

Dans cette section, nous présentons le modèle PERSUA2 que nous proposons pour définir une personnalisation des activités. Nous expliquons tout d'abord les principes de ce modèle et de son processus d'exploitation. Nous donnons ensuite la définition formelle de PERSUA2 avant d'illustrer son utilisation à l'aide du scénario présenté dans la section 2.1 Nous terminons en présentant l'architecture du processus d'exploitation du modèle permettant de créer des séquences de travail personnalisées. 


\subsection{Principe du modèle PERSUA2}

Pour aider l'enseignant à personnaliser les activités d'apprentissage qu'il propose, il est nécessaire de connaître les critères d'individualisation qu'il souhaite mettre en œuvre. Ces critères portent d'une part sur la manière dont il souhaite affecter une activité à un apprenant, et d'autre part sur le contexte dans lequel les apprenants vont utiliser la séquence de travail qui leur est destinée.

Le modèle PERSUA2 (PERSonnalisation Unifiée des Activités d'Apprentissage) que nous proposons comprend deux éléments (cf. Figure 3) : la stratégie pédagogique à mettre en place, c'est-à-dire les règles permettant d'affecter une activité à un apprenant, et le contexte d'utilisation de cette stratégie pédagogique.

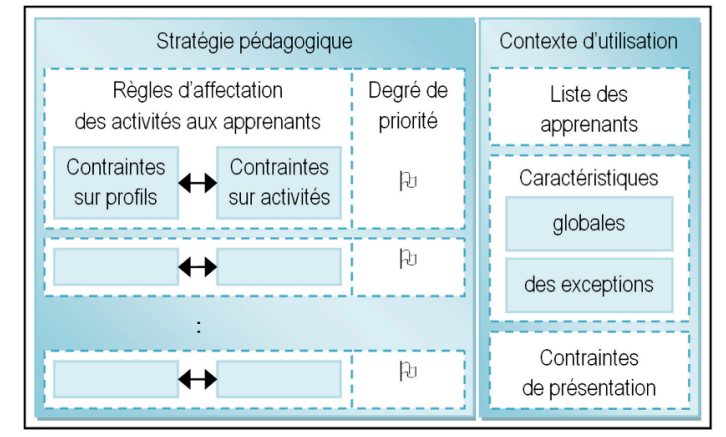

Figure 3 • Principe du modèle PERSUA2

Une stratégie pédagogique définit comment affecter des activités aux apprenants. Cette affectation est décrite à l'aide d'un ensemble de liens appelés règles d'affectation. Une règle d'affectation lie des contraintes sur le profil des apprenants à des contraintes sur une ou plusieurs activités pédagogiques. Les contraintes sur le profil permettent de sélectionner certaines valeurs du profil des apprenants. Les contraintes sur les activités permettent de sélectionner une activité correspondant à la stratégie pédagogique à mettre en place. Par activité, nous entendons d'une part des exercices papier à imprimer, et d'autre part des activités sur un logiciel pédagogique associées à la configuration de l'environnement du logiciel. Un degré de priorité est associé à chaque règle d'affectations afin de pouvoir choisir la règle la plus importante quand plusieurs règles peuvent s'appliquer. 
Un contexte d'utilisation définit quant à lui un ensemble d'informations permettant de caractériser la situation dans laquelle se trouve un apprenant lorsque qu'il effectue la séance de travail. Ces informations portent, entre autres, sur la liste des élèves pour lesquels une personnalisation des séances est souhaitée, sur leur profil, mais aussi sur des caractéristiques plus générales de la séquence d'activités (nombre d'exercices, durée, etc.). Le contexte contiendra, en plus de ces informations globales à tous les apprenants, des exceptions pour certains d'entre eux.

L'indépendance des deux parties du modèle de personnalisation permet d'associer une même stratégie pédagogique à plusieurs contextes d'utilisation et inversement. Ainsi l'enseignant peut définir une unique stratégie de personnalisation qui explicite comment il estime souhaitable d'affecter les activités, et l'utiliser pour des séances qui n'ont pas toutes la même durée et ne concernent pas les mêmes élèves à chaque fois. Inversement, pour un même groupe d'élèves et des séances de durées identiques, l'enseignant pourra choisir d'utiliser des stratégies de personnalisation différentes, selon le contenu sur lequel il veut travailler pour une séance donnée, chacune pouvant refléter un objectif pédagogique différent.

La mise en œuvre de ce modèle PERSUA2 permet à un enseignant de définir son propre modèle de personnalisation. Pour que ce modèle puisse être utilisé, nous lui avons associé un processus d'exploitation dont le principe est le suivant :

- L'enseignant définit des règles d'affectation des activités aux apprenants

- en contraignant le profil d'apprenant pour en sélectionner une partie, puis en contraignant les valeurs que les profils d'apprenants contiendront pour cette partie (cf. (1) sur la Figure 4),

- en contraignant le choix ou la génération des activités à fournir (cf. (2) sur la Figure 4).

- L'enseignant hiérarchise ensuite ces règles d'affectation en fonction de leur niveau de priorité pour créer sa stratégie pédagogique (cf. (3) sur la Figure 4).

- L'enseignant définit un contexte d'utilisation de sa stratégie pédagogique contenant, entre autres, la liste des apprenants pour lesquels il souhaite une séquence personnalisée (cf. les deux points (4) sur la Figure 4).

- Le système filtre les règles d'affectation pour ne garder, pour chaque apprenant, que celles dont les prémisses sont vérifiées par les valeurs de son profil (cf. (5) sur la Figure 4). 
- Le système construit une liste d'activités à générer à partir des règles d'affectation retenues et des contraintes du contexte (temps, nombre d'exercices...) (cf. (6) sur la Figure 4).

- Le système crée les sessions personnalisées en fonction des listes d'activités à générer (cf. (7) sur la Figure 4).

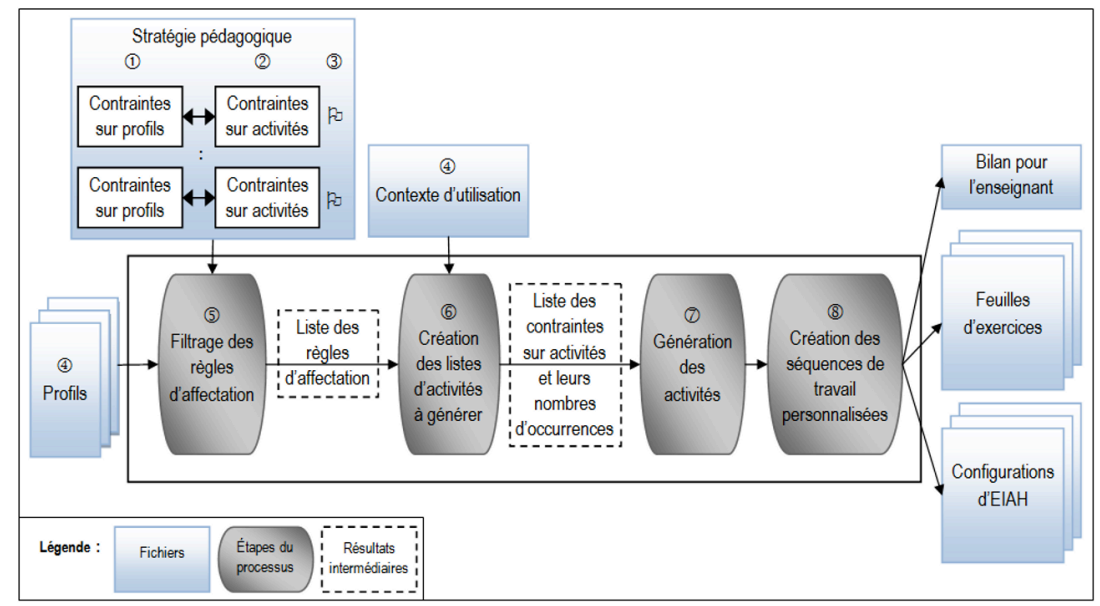

Figure 4 • Principe du modèle PERSUA2 et de son processus d'exploitation

En respectant ce processus d'exploitation, le système crée autant de séances de travail personnalisées qu'il y a de profils d'élèves, ainsi qu'un bilan pour l'enseignant (cf. 8) sur la Figure 4). La création des séances de travail se fait soit en générant des activités papier, soit en configurant des logiciels pédagogiques existants.

\subsection{Définition formelle du modèle PERSUA2}

Nous venons de voir les principes de notre modèle de personnalisation PERSUA2. Nous en donnons maintenant une définition formelle (cf. Figure 5). 


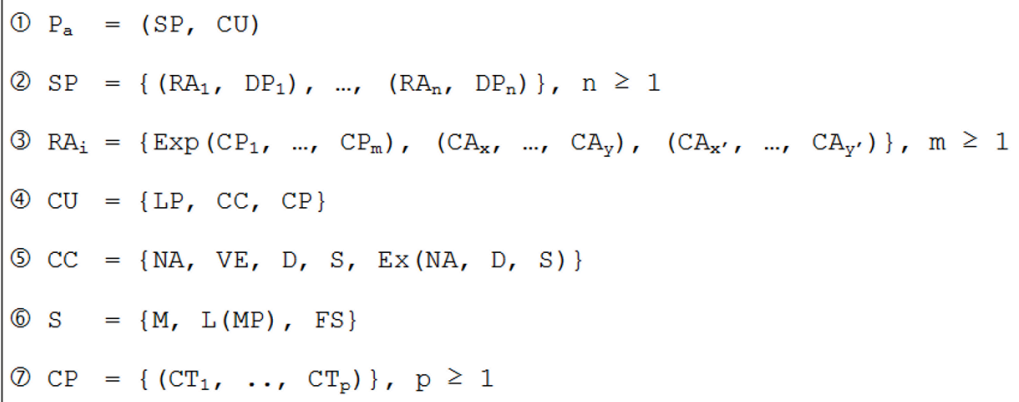

Figure 5 • Définition formelle du modèle PERSUA2

Le modèle de la personnalisation d'une séquence de travail voulue par un agent $a$ est noté $P_{a}$ (cf. (1) sur la Figure 5). L'agent $a$ est soit un enseignant, soit un EIAH. Le modèle de personnalisation $P_{a}$ peut être appliqué à un apprenant ou à un ensemble d'apprenants. Il se compose d'une stratégie pédagogique $S P$ et d'un contexte d'utilisation $C U$.

Une stratégie pédagogique $S P$ (cf. (2) sur la Figure 5) est un ensemble de paires constituées d'une règle d'affectation $R A$ et de son degré de priorité $D P$. Une stratégie pédagogique $S P$ peut contenir autant de paires $\left(R A_{i}, D P_{i}\right)$ que nécessaire. Le degré de priorité $D P_{i}$ d'une règle d'affectation $R A_{i}$ est une valeur appartenant à une liste graduée, définie par une borne inférieure, une borne supérieure et un pas.

Une règle d'affectation $R A_{i}$ (cf. (3) sur la Figure 5) est un triplet composé d'une expression booléenne portant sur des contraintes sur les profils $C P_{j}$ et de deux listes de contraintes sur les activités $C A_{k}$. Ce triplet représente les conditions et les conclusions d'une règle de type «si-alors ». L'équation (3) est donc équivalente à l'équation suivante :

$\mathrm{RA}_{i}=\mathrm{SI} \operatorname{Exp}\left(\mathrm{CP}_{1}, \ldots, C \mathrm{P}_{\mathrm{m}}\right) \operatorname{ALORS}\left(\mathrm{CA}_{\mathrm{x}}, \ldots, \mathrm{CA}_{\mathrm{y}}\right)$ SINON $\left(\mathrm{CA}_{\mathrm{x}^{\prime}}, \ldots, \mathrm{CA}_{\mathrm{y}^{\prime}}\right)$

Les contraintes sur les profils $C P_{j}$ ont été formalisées à partir du langage de description de profils PMDLe (Eyssautier-Bavay, 2008) (Jean-Daubias et Ginon, 2010). Le modèle cPMDLe des contraintes $C P_{j}$ et les différentes façons de les combiner pour construire une expression booléenne sont définis dans (Lefevre, 2009). La première liste de contraintes sur activités $\left(C A_{x}, \ldots, C A_{y}\right)$ correspond aux contraintes permettant 
de créer les activités qui seront fournies à un apprenant dont les valeurs du profil respecteront l'expression booléenne. La seconde liste $\left(C A_{x^{\prime}}, \ldots, C A_{y^{\prime}}\right)$, qui est optionnelle, correspond aux contraintes permettant de créer les activités qui seront fournies si les valeurs du profil ne respectent pas l'expression. L'intersection entre les listes $\left(C A_{x}, \ldots, C A_{y}\right)$ et $\left(C A_{x^{\prime}}, \ldots, C A_{y^{\prime}}\right)$ peut être non vide, c'est-à-dire qu'un même ensemble de contraintes pourra être utilisé pour créer une activité qui sera fournie à des apprenants dont les valeurs du profil respectent ou non l'expression booléenne. La formalisation des contraintes sur activités $C A_{k}$ respecte les principes de l'approche GEPPETO (Lefevre 2010).

Un contexte d'utilisation $C U$ (cf. (4) sur la Figure 5) contient la liste des profils d'apprenants $L P$, un ensemble de contraintes de création des séquences d'activités $C C$ et un ensemble de contraintes de présentation $C P$.

La liste des profils d'apprenants $L P$ permet de connaître les apprenants concernés par la personnalisation. Les contraintes de création $C C$ permettent de fixer des limites générales pour la création des séquences d'activités, et les contraintes de présentation $C P$ permettent de fixer des conditions de présentation des activités au sein des séquences. Les contraintes $C C$ et $C P$ du contexte d'utilisation sont appliquées pour les séquences de tous les apprenants contenus dans $L P$.

Les contraintes de création $C C$ (cf. (5) sur la Figure 5) portent sur le nombre d'activités $N A$, la variation des énoncés $V E$, la durée de la séance $D$, le support de la séquence $S$ et les exceptions $E x$. Ces contraintes vont intervenir lors de la création des séquences de travail personnalisées.

Le nombre d'activités $N A$ est un intervalle $\left[N A_{\min }, N A_{\max }\right]$ permettant de spécifier un nombre d'activités minimum et un nombre d'activités maximum que doit contenir la séquence de travail.

La variation des énoncés $V E$ permet d'indiquer, dans le cas où plusieurs apprenants doivent faire la même activité, si l'instanciation de l'activité doit être la même pour tous les apprenants, ou différente pour chaque apprenant. Cette contrainte peut être utilisée pour fournir, par exemple, une évaluation où les énoncés d'exercices sont différents d'un élève à l'autre.

La durée de la séance $D$ est un intervalle $\left[D_{\min }, D_{\max }\right]$ permettant de spécifier une durée minimum et une durée maximum pour la séquence de travail. Cette contrainte sert par exemple à adapter la durée de la séquence au temps dont dispose 
un enseignant pour faire travailler ses élèves en soutien ou pour fournir un devoir à la maison personnalisé d'une certaine durée

Le support d'une séquence d'activités $S$ (cf. (6) sur la Figure 5) précise les contraintes techniques pour créer la séquence travail.

La première contrainte concerne le matériel $M$ dont disposent les apprenants pour effectuer la séquence de travail (papier, ordinateur ou les deux). Si cette contrainte indique que les apprenants travaillent sur papier, d'autres contraintes sont alors disponibles : la longueur de la séquence $L$, la mise en page $M P$ et le format de sortie $F S$.

La longueur de la séquence $L$ est un intervalle $\left[L_{\min }, L_{\max }\right]$ permettant de définir une longueur physique pour la page à imprimer à l'aide d'un minimum et d'un maximum. Cette longueur physique dépend des contraintes de mise en page $M P$.

Les contraintes de mise en page $M P$ permettent de spécifier la mise en forme des caractères : police, taille, style, etc.

Le format de sortie $F S$ permet de préciser le format de la feuille d'exercices à imprimer (RTF, HTML...).

Les exceptions $E x$ permettent de définir des contraintes propres à certains apprenants dont les profils sont fournis dans $L P$. Ces exceptions peuvent porter sur le nombre d'activités $N A$, la durée de la séance $D$, mais aussi le support de la séquence d'activité $S$. Elles permettent de prendre en compte les handicaps temporaires d'un apprenant, leurs contraintes d'emploi du temps, etc. Un enseignant pourra par exemple faire travailler tous ses élèves durant la dernière heure de cours de la journée, sauf ceux qui doivent partir en soutien la dernière demi-heure.

Les contraintes de création sont toutes optionnelles et peuvent être combinées. Il est par exemple possible de demander une séquence d'exercices sur papier (M) contenant entre trois et cinq exercices (NA) pour une durée totale de trente minutes (D). Dans cet exemple, les contraintes VE, L, MP, FS ne sont pas définies et aucune exception n'est précisée. Pour ces contraintes non définies par l'agent de personnalisation, les séquences d'activités seront créées à partir des valeurs par défaut propres au système mettant en œuvre cette personnalisation. Par exemple, un premier système pourra proposer des fichiers PDF à imprimer tandis qu'un autre fournira des feuilles au format HTML. 
Les contraintes de présentation $C P$ (cf. (7) sur la Figure 5) portent sur l'ordonnancement des activités dans la séquence de travail. Elles ne modifient pas le contenu d'une séquence, mais permettent de classer les activités en fonction de certains critères de tri $C T_{k}$. Les critères portent sur les métadonnées des activités contenues dans la séance.

La Figure 6 reprend la Figure 3 en situant les différentes parties du modèle formel présentées dans cette section.

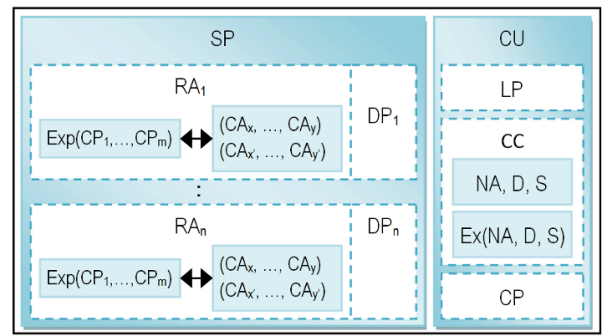

Figure 6 • Représentation graphique du modèle PERSUA2

\subsection{Exemple d'utilisation du modèle PERSUA2}

Pour illustrer 1'utilisation du modèle PERSUA2, reprenons le premier scénario d'usage présenté au début de l'article (cf. section 2.1.). Dans ce scénario, Dimitri, enseignant de CE2, souhaite s'appuyer sur les informations dont il dispose sur les connaissances des six élèves concernés par le soutien pour proposer des séances de remédiation adaptées à chacun.

Les séances de soutien ont lieu dans une salle combinant une partie classique, avec un tableau et des tables, et une partie informatique, avec des ordinateurs disposant notamment d'un logiciel d'entraînement aux tables d'addition et de multiplication. Les activités proposées aux élèves peuvent donc être soit papier, soit logicielles.

Pour préparer ces séances, Dimitri a créé, pour chacun des six élèves, un profil contenant des informations sur leurs compétences en français et en mathématiques. À titre d'exemple, la Figure 7 montre les profils de Jean et de Léa. 


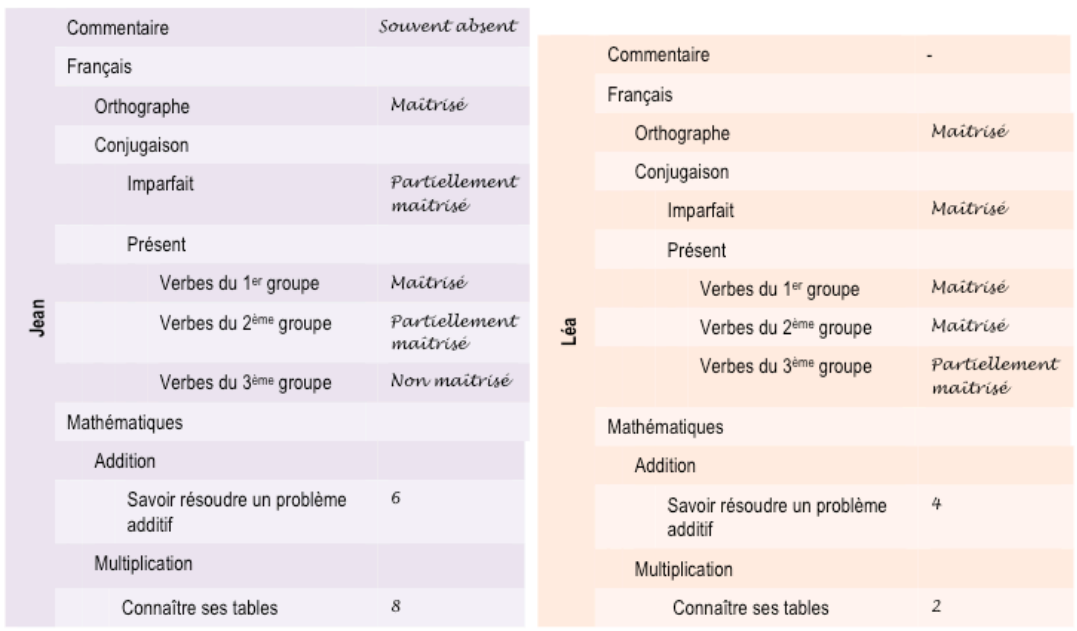

Figure 7 • Profils de Jean et de Léa

Pendant ces séances de soutien, Dimitri veut faire travailler les élèves qui ne mâ̂trisent pas les compétences de conjugaison sur un exercice papier de type P_B17 $7^{1}$ et ceux qui les maîtrisent partiellement sur deux exercices papier, l'un de type P_B18a et l'autre de type P_B18b. Cela revient à regarder la valeur de l'élément « Français Conjugaison » dans le profil de l'élève, élément qui prend pour valeur la moyenne de ses sous-éléments. L'échelle associée à cette valeur est la liste énumérée « non maitrisé, partiellement maîtrisé, maîtrisé ». Si la valeur de l'élève est « non maîtrisé », alors l'élève aura un exercice papier de type P_B17, si la valeur est « partiellement maîtrisé », alors l'élève aura un exercice papier de type P_B18a et un exercice papier de type P_B18b, enfin si la valeur est « maîtrisé », l'élève n'aura pas d'exercice.

Dimitri souhaite également faire travailler les élèves sur les problèmes additifs. Il souhaite que les élèves ne maitrisant pas cette compétence travaillent sur un exercice papier de type P_C24a, que ceux la maîtrisant partiellement travaillent sur un exercice papier de type P_C24b et un de type P_C24c et que ceux la maîtrisant travaillent sur un exercice papier de type $\mathrm{P}_{-} \mathrm{C} 24 \mathrm{~d}$. Cela revient à regarder la valeur de l'élément «Mathématiques - Addition - Savoir résoudre un problème additif» dans le profil de l'élève. Cette valeur est définie grâce à un entier allant de 0 à 10 . Si la valeur de l'élève est strictement inférieure à 5 alors l'élève aura un exercice papier de type P_C24a, si la valeur est comprise entre 5 inclus et 8 exclu, alors l'élève aura un exer- 
cice papier de type $\mathrm{P}_{-} \mathrm{C} 24 \mathrm{~b}$ et un de type $\mathrm{P} \_\mathrm{C} 24 \mathrm{c}$, sinon l'élève aura un exercice papier de type $\mathrm{P} \_\mathrm{C} 24 \mathrm{~d}$.

Dimitri souhaite enfin faire travailler les élèves sur la multiplication en utilisant le logiciel installé dans la salle informatique où il se trouve. Il souhaite que les élèves ne maîtrisant pas la multiplication fassent une activité de type S_A12 sur le logiciel et que les autres fassent une activité de type S_A75, toujours sur le logiciel. Cela revient à regarder la valeur de l'élément « Mathématiques - Multiplication » dans le profil de l'élève, élément qui prend la valeur de son seul sous-élément. Cette valeur est définie grâce à un entier allant de 0 à 10 . Si la valeur pour l'élève est strictement inférieure à 5, il aura une activité logicielle de type S_A12 sur le logiciel de multiplication, dans le cas contraire il aura une activité logicielle de type S_A75 sur le même logiciel.

Pour la prochaine séance de soutien, Dimitri souhaite faire travailler ses élèves en priorité sur la conjugaison, puis sur la multiplication. Il veut de plus que chacun des élèves fasse entre trois et quatre activités.

Cette stratégie de création de séances de soutien personnalisées peut être formalisée grâce au modèle PERSUA2. Cette formalisation est fournie dans la Figure 8 et peut être schématisée par la Figure 9. Comme nous le verrons dans la section 6 de cet article, décrivant la mise en œuvre du modèle PERSUA2, la façon dont l'enseignant explicitera sa stratégie de personnalisation est plus proche de la schématisation de la Figure 9 que de la formalisation de la Figure 8. 


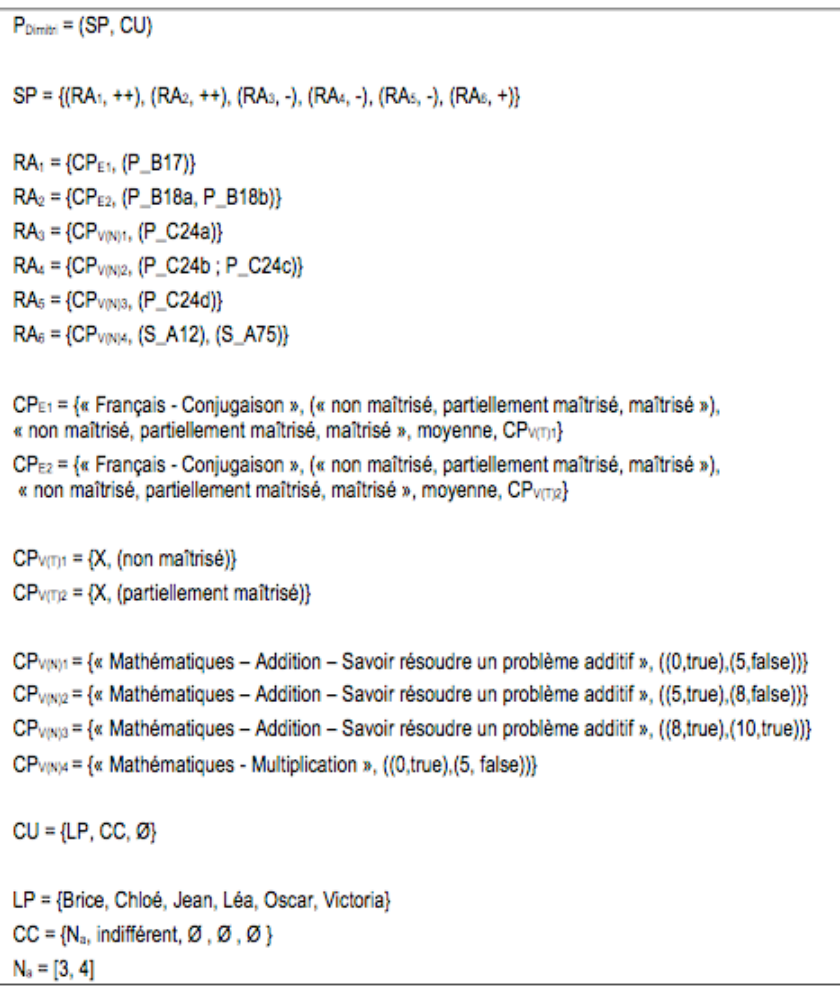

Figure 8 • Exemple d'un modèle de personnalisation

\begin{tabular}{|c|c|c|c|c|}
\hline \multicolumn{5}{|c|}{ Stratégie pédagogique } \\
\hline ID & SI & ALORS & SINON & Po \\
\hline$R A_{1}$ & Conjugaison = [Non maîtrisé $]$ & Activité papier type P_B17 & & ++ \\
\hline $\mathrm{RA}_{2}$ & Conjugaison $=[$ Partiellement maîtrisé $]$ & Activité papier type $\mathrm{P} \_$B18a et $\mathrm{P} \_$B18b & & ++ \\
\hline $\mathrm{RA}_{3}$ & Addition- Problème additif $=[0,5[$ & Activité papier type $P_{-}$C24a & & - \\
\hline $\mathrm{RA}_{4}$ & Addition- Problème additif $=[5,8[$ & Activité papier type P_C24b et P_C24c & & - \\
\hline $\mathrm{RA}_{5}$ & Addition- Problème additif $=[8,10]$ & Activité papier type $P_{-} C 24 d$ & & - \\
\hline $\mathrm{RA}_{6}$ & Multiplication $=[0,5[$ & Activité logicielle type S_A12 & Activité logicielle type S_A75 & + \\
\hline \multicolumn{5}{|c|}{ Contexte d'utilisation } \\
\hline \multicolumn{2}{|c|}{ Liste des élèves } & \multicolumn{3}{|l|}{ Brice, Chloé, Jean, Léa, Oscar, Victoria } \\
\hline \multicolumn{2}{|c|}{ Nombre d'exercices } & \multicolumn{3}{|l|}{$\operatorname{Min}=3 ; \operatorname{Max}=4$} \\
\hline
\end{tabular}

Figure 9 • Schématisation du modèle de personnalisation de la Figure 8 
Pour exploiter ce modèle de personnalisation, une première étape consiste à comparer les valeurs contenues dans les profils des élèves avec les conditions des règles d'affectation définies par l'enseignant afin d'obtenir la liste des règles activées par le profil, ainsi que la liste des types d'activités concernées pour chaque règle.

Illustrons cette exploitation à l'aide du profil de Jean (cf. partie gauche de la Figure 7).

Pour interpréter les règles d'affectation $\mathrm{RA}_{1}$ et $\mathrm{RA}_{2}$, il faut obtenir la valeur de l'élément « Français - Conjugaison» en faisant une moyenne des valeurs de ses souséléments. On fait tout d'abord la moyenne de l'élément «Français - Conjugaison Présent » qui a pour résultat «partiellement maîtrisé ». Puis on fait la moyenne des éléments «Français - Conjugaison - Présent» et «Français - Conjugaison - Imparfait » qui a pour résultat «partiellement maîtrisé ». Jean a donc la valeur «partiellement maîtrisé » pour l'élément «Français - Conjugaison ». On retient donc la règle d'affectation $\mathrm{RA}_{2}$.

Pour interpréter les règles d'affectation $\mathrm{RA}_{3}, \mathrm{RA}_{4}$ et $\mathrm{RA}_{5}$, il faut exploiter l'élément « Mathématiques - Addition - Savoir résoudre un problème additif ». Jean a 6 pour cet élément. Or 6 appartient seulement à l'intervalle d'application de la règle d'affectation $\mathrm{RA}_{4}([5,8])$. On retient donc uniquement la règle $\mathrm{RA}_{4}$.

Pour interpréter la règle $\mathrm{RA}_{6}$, il faut exploiter l'élément « Mathématiques - Multiplication». Jean a 8 pour cet élément (note de l'unique sous-élément). Or 8 n'appartient pas à l'intervalle [0,5[. La règle d'affectation $\mathrm{RA}_{6}$ ayant deux listes de types d'activités, une pour les élèves validant la condition de la règle et une pour ceux ne la validant pas. On retient donc la règle $\mathrm{RA}_{6}$ avec la seconde liste de types d'activités.

La synthèse de cette interprétation des règles d'affectation en fonction des valeurs du profil de Jean est fournie sur la partie gauche de la Figure 10. 


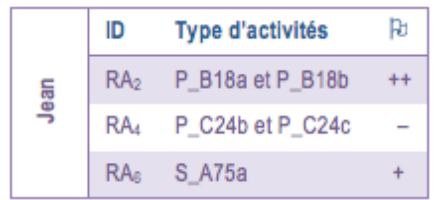

\begin{tabular}{|l|lll|}
\hline \multirow{2}{*}{$\Xi$} & ID & Type d'activités & Pu \\
\cline { 2 - 4 } & RA4 & P_C24a & - \\
& RA 4 & S_A12 & + \\
\hline
\end{tabular}

Figure 10 • Listes des règles d'affectation, et des types d'activités associés, activées par le profil de Jean (partie gauche) et par le profil de Léa (partie droite)

Prenons maintenant le profil de Léa (cf. partie droite de la Figure 7).

Pour interpréter les règles d'affectation $\mathrm{RA}_{1}$ et $\mathrm{RA}_{2}$, il faut obtenir la valeur de l'élément « Français - Conjugaison» en faisant une moyenne. On fait tout d'abord la moyenne de l'élément « Français - Conjugaison - Présent » qui a pour résultat « maîtrisé ». Puis on fait la moyenne des éléments « Français - Conjugaison - Présent » et «Français - Conjugaison - Imparfait » qui a pour résultat «maîtrisé ». Léa a donc la valeur «maîtrisé » pour l'élément «Français - Conjugaison ». On ne retient donc aucune des deux règles d'affectation pour cette élève.

Pour interpréter les règles d'affectation $\mathrm{RA}_{3}, \mathrm{RA}_{4}$ et $\mathrm{RA}_{5}$, il faut exploiter l'élément « Mathématiques - Addition - Savoir résoudre un problème additif ». Léa a une note de 4 pour cet élément et valide donc la condition de la règle d'affectation $\mathrm{RA}_{3}$.

Pour interpréter la règle d'affectation $\mathrm{RA}_{6}$, il faut exploiter l'élément « Mathématiques - Multiplication ». Léa a une note 2 pour cet élément. On retient donc la règle $\mathrm{RA}_{6}$ avec la première liste de types d'activités.

La synthèse de cette interprétation des règles d'affectation en fonction des valeurs du profil de Léa est fournie sur la partie droite de la Figure 10.

À partir des deux listes contenant, pour chaque élève, la liste des types d'activités à générer, il est possible de prendre en compte le contexte d'utilisation représenté dans le modèle de personnalisation pour obtenir la liste définitive des activités à générer pour chaque élève.

Prenons la liste des activités à générer pour Jean (cf. partie gauche de la Figure 10). Le résultat de l'application des règles d'affectation sur son profil donne cinq activités à générer (de type P_B18a, P_B18b, P_C24b, P_C24c, S_A75). Or le contexte d'utilisation spécifie qu'il en faut au maximum quatre. Il faut donc en supprimer 
une. C'est à ce moment là que le niveau de priorité de la règle d'affectation entre en ligne de compte. On va supprimer de la liste l'activité à générer associée à la règle d'affectation de plus faible niveau de priorité, soit la règle $\mathrm{RA}_{4}$. Cette règle étant associée à deux types d'activités, on en supprime une choisie aléatoirement, soit l'activité à générer $\mathrm{P} \_\mathrm{C} 24 \mathrm{~b}$. Nous avons donc maintenant une liste d'activités à générer correspondant à la fois au profil de Jean, à la stratégie pédagogique de l'enseignant et compatible avec le contexte d'utilisation (cf. partie gauche de la Figure 11).

Prenons maintenant la liste des activités à générer pour Léa (cf. partie droite de la Figure 10). Il y a pour l'instant deux activités à générer (de type P_C24a et S_A12), or le contexte d'utilisation spécifie qu'il en faut au minimum trois. Il faut donc en ajouter une. On va ajouter l'activité à générer associée à la règle d'affectation de plus haute priorité, soit la règle $\mathrm{RA}_{6}$ et l'activité à générer de type $\mathrm{S}_{-} \mathrm{A} 12$. Nous avons donc maintenant une liste d'activités à générer correspondant à la fois au profil de Léa, à la stratégie pédagogique de l'enseignant et compatible avec le contexte d'utilisation (cf. partie droite de la Figure 11).

\begin{tabular}{|c|c|c|c|c|c|}
\hline \multirow{6}{*}{ 䓌 } & Type d'activité & Occurrence & & & \\
\hline & P_B18a & 1 & & & \\
\hline & P_B18b & 1 & & & \\
\hline & P_C24b & 0 & \multirow{3}{*}{$\stackrel{\Xi}{\Xi}$} & Type d'activités & Occurrence \\
\hline & P_C24C & 1 & & P_C24b & 1 \\
\hline & S_A75 & 1 & & S_A12 & 2 \\
\hline
\end{tabular}

Figure 11 • Liste des activités à générer pour Jean (partie gauche) et Léa (partie droite)

Maintenant que nous avons pour chaque élève la liste des activités à générer, il reste à les générer et à créer, à partir des résultats de la génération, les feuilles d'exercices à imprimer et les sessions sur le logiciel de conjugaison. Pour les activités papier, il est nécessaire de posséder des générateurs d'exercices, ainsi que des connaissances permettant de combiner ces exercices afin de créer une feuille d'exercices à imprimer. Pour les activités sur le logiciel de multiplication, un modèle respectant l'approche GEPPETO et décrivant ce logiciel permet d'une part de générer une activité ou de la choisir dans la base d'activités du système en fonction des contraintes spécifiées par l'enseignant, et d'autre part de créer les fichiers de configuration du logiciel permettant de le paramétrer pour chaque élève. Dans les deux cas, le modèle 
PERSUA2 s'appuie sur l'approche GEPPETO (Lefevre, 2009), (Lefevre, 2010) afin de permettre cette création d'activités en fonction des choix de l'enseignant.

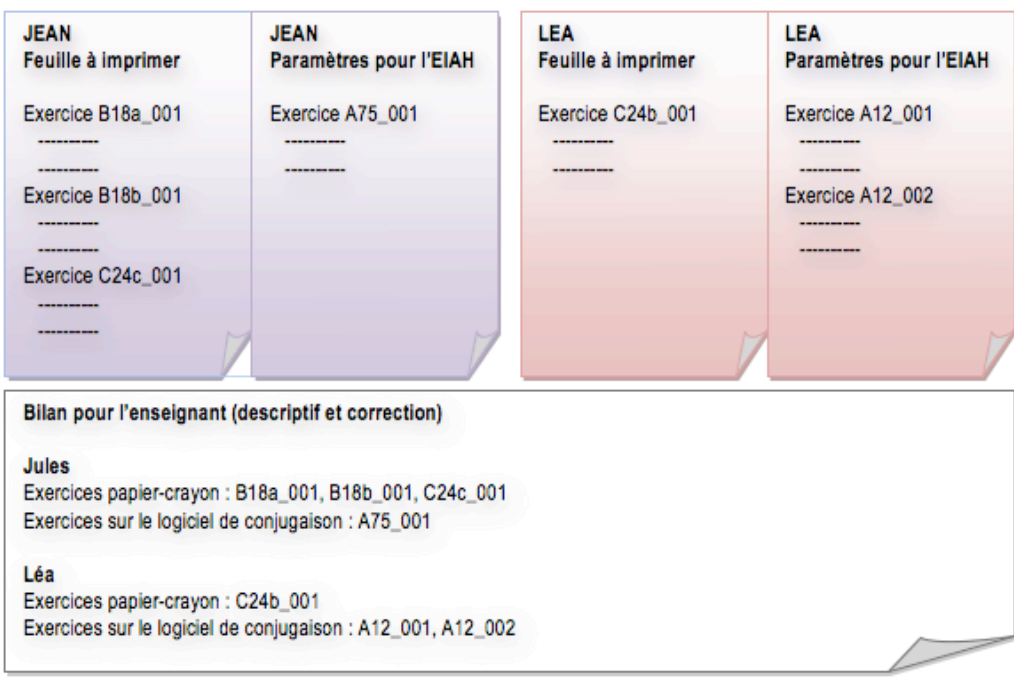

Figure 12 • Résultat du processus de création de séquences d'activités personnalisées

À la fin de cette étape de génération des activités et de création des séquences de travail pour chaque élève, nous obtenons (cf. Figure 12) :

- Pour Jean :

- une feuille d'exercices à imprimer contenant trois exercices respectant les types P_B18a, P_B18b et P_C24c ;

- un fichier de configuration pour le logiciel de multiplication permettant de faire une activité de type S_A75.

- Pour Léa :

- une feuille d'exercices à imprimer contenant un exercice de type P_C24b ;

- un fichier de configuration pour le logiciel de multiplication permettant de faire deux activités de type S_A12. Ces activités ne possèdent pas le même énoncé, mais possèdent des caractéristiques en commun (par exemple, le nombre de tables à connaître, ou des tables précises comme celle de 9).

- Pour l'enseignant :

- un bilan contenant un descriptif des activités fournies à chacun de ses élèves, ainsi que la correction des activités des feuilles d'exercices papier. 


\subsection{Architecture du processus de génération de séquences personna- lisées}

Nous détaillons à présent formellement l'architecture du processus d'exploitation du modèle PERSUA2 permettant la génération de séquences personnalisées.

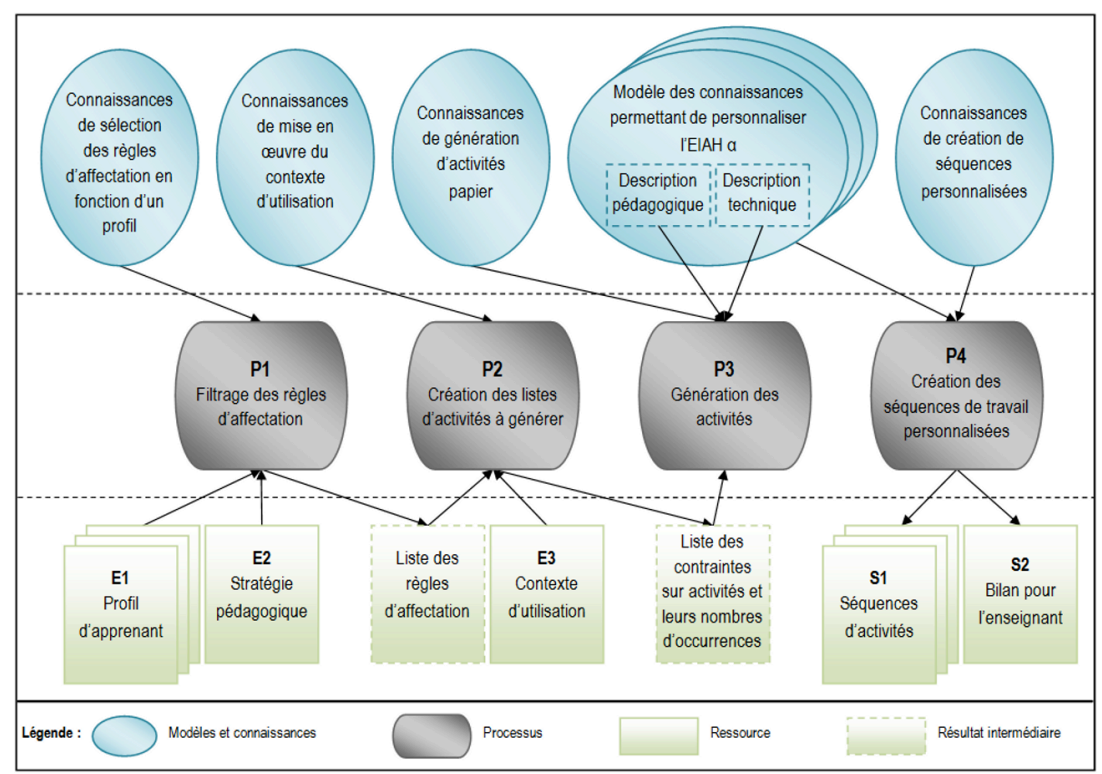

Figure 13 • Architecture du processus de génération de séquences d'activités personnalisées

Le processus prend en entrée trois éléments : les profils des apprenants (cf. E1 sur la Figure 13), la stratégie pédagogique de l'enseignant (cf. E2 sur la Figure 13) et le contexte d'utilisation des séquences d'activités personnalisées (cf. E3 sur la Figure 13). Il fournit en sortie deux types d'éléments : les séquences d'activités adaptées au profil de chaque apprenant (cf. S1 sur la Figure 13) et un bilan des propositions à destination de l'enseignant (cf. S2 sur la Figure 13).

Ce processus est constitué de quatre parties : le filtrage des règles d'affectation (cf. P1 sur la Figure 13), la création des listes d'activités à générer (cf. P2 sur la Figure 13), la génération des activités (cf. P3 sur la Figure 13) et la création de sé- 
quences de travail (cf. P4 sur la Figure 13). Nous présentons, dans la suite de cette section, chacun de ces constituants.

\subsubsection{Filtrage des règles d'affectation}

Le processus de filtrage (cf. P1 sur la Figure 13) permet de sélectionner les règles d'affectation dans lesquelles les conditions sont validées par les valeurs contenues dans le profil d'un apprenant.

Pour décrire les profils d'apprenant, nous avons choisi d'utiliser le formalisme PMDLe (Eyssautier-Bavay, 2008) (Jean-Daubias et Ginon, 2010). Les profils contiennent des données potentiellement de toutes disciplines et de tous niveaux, issues de profils papier-crayon venant des pratiques en classe des enseignants, mais également de profils établis par des logiciels.

La stratégie pédagogique respectant le modèle PERSUA2 rassemble un ensemble de règles d'affectation. Ces règles d'affectation sont des liens entre des éléments du profil des apprenants et des structures d'activités contenant un ensemble de contraintes permettant de générer ou de choisir une activité.

Les connaissances de sélection des règles d'affectation en fonction d'un profil permettent d'interpréter les règles d'affectation représentées dans les stratégies pédagogiques en fonction des valeurs contenues dans les profils d'apprenants. Ces connaissances contiennent d'une part des règles permettant de savoir si les valeurs contenues dans le profil respectent les conditions des règles d'affectation des stratégies pédagogiques et d'autre part des formules permettant de convertir les valeurs $\mathrm{du}$ profil dans une échelle donnée.

Le filtrage des règles d'affectation prend en entrée la stratégie pédagogique de l'enseignant et un ensemble de profils d'apprenants. Pour chaque profil, les conditions des règles d'affectation vont être évaluées pour savoir si elles sont validées par les valeurs contenues dans le profil. Le cas échéant, les règles d'affectation sont ajoutées à la liste des règles applicables pour l'apprenant. À la fin du filtrage, on obtient autant de listes de règles d'affectation que l'on avait de profils d'apprenants en entrée.

\subsubsection{Création des listes d'activités à générer}

Le processus de création des listes d'activités à générer (cf. P2 sur la Figure 13) permet, pour chaque apprenant, de définir les activités qui doivent être générées afin qu'il travaille dans les conditions précisées dans le contexte d'utilisation. 
Le contexte d'utilisation permet à l'enseignant de préciser des contraintes permettant de «borner » les feuilles d'exercices ou les sessions sur les EIAH, c'est-à-dire donner une durée pour la séance de travail, un nombre maximum d'exercices, etc. Le contexte d'utilisation respecte le modèle PERSUA2.

Les connaissances de mise en œuvre du contexte d'utilisation permettent d'interpréter les contraintes contenues dans le contexte d'utilisation et de les mettre en relation avec les règles d'affectation concernées par un profil. Elles permettent de déterminer le nombre d'occurrences nécessaire pour chacune des contraintes sur activités contenues dans les règles d'affectation, afin de respecter les contraintes du contexte d'utilisation. Ces connaissances sont exprimées par des règles permettant de dupliquer ou de supprimer les activités à générer contenues dans les règles d'affectation pour obtenir un nombre d'activités à générer ou une durée de travail correspondant aux contraintes du contexte d'utilisation.

La création des listes d'activités à générer prend en entrée les règles d'affectation concernées par un profil, ainsi que le contexte d'utilisation, et retourne la liste des activités à générer pour créer la séquence de travail d'un apprenant. Un nombre d'occurrences est associé à ces activités pour respecter les contraintes de temps, de nombre, etc. du contexte d'utilisation. À la fin de cette étape, on obtient autant de listes d'activités à générer que l'on avait de listes de règles d'affectation et donc de profils d'apprenants.

\subsubsection{Génération des activités}

Le processus de génération d'activités (cf. P3 sur la Figure 13) permet de choisir ou de générer l'énoncé de l'activité, ainsi que sa correction en ce qui concerne les activités papier.

Les connaissances de génération d'activités papier contiennent des patrons d'exercices associés à des générateurs, respectant tous l'approche GEPPETO (Lefevre, 2010).

Les différents modèles des connaissances $\mathrm{OKEP} / \mathrm{x}^{2}$ permettant la personnalisation des EIAH x sont des instances du méta-modèle $\mathrm{AKEPI}^{3}$. Ce méta-modèle, issu de l'approche GEPPETO (Lefevre et al., 2009), permet à des experts de définir les connaissances nécessaires à la personnalisation des EIAH. Nous appelons "expert», pour chaque EIAH à prendre en charge, le concepteur de l'EIAH ou toute personne connaissant suffisamment le logiciel pour le décrire d'un point de vue pédagogique et 
d'un point de vue technique. Cette expertise peut être apportée par plusieurs personnes, chacun ayant sa spécialité (le développeur du logiciel, un pédagogue, etc.). Chaque modèle OKEP/x définit par un expert contient donc la description pédagogique et la description technique de l'EIAH x. La description pédagogique contient la liste des propriétés personnalisables de l'EIAH, ainsi que les règles qui permettent de les gérer. La description technique regroupe toutes les informations nécessaires pour agir concrètement sur le système: localisation du système, des générateurs d'exercices ou de la base d'exercices, place et contenu des fichiers de configuration et règles permettant de remplir ces fichiers.

La génération des activités prend en entrée la liste des activités à générer, ainsi que leur nombre d'occurrences. Pour chaque activité à générer, le processus génère le nombre d'activités demandées en vérifiant éventuellement que l'énoncé de chaque occurrence est unique et en s'assurant, dans l'historique de chaque apprenant, que l'activité générée ne lui a pas déjà été proposée. Pour générer les activités papier, le système se sert des générateurs d'exercices contenus dans les connaissances de génération d'activités papier. Pour les activités sur des EIAH externes, le système se sert du ou des générateurs contenus dans l'EIAH quand ceux-ci existent et sont utilisables via des fichiers de configuration; dans le cas contraire, il choisit les activités dans la base de données de l'EIAH.

\subsubsection{Création des séquences de travail personnalisées}

Le processus de création des séquences de travail personnalisées (cf. P4 sur la Figure 13) permet de combiner les activités pour créer des feuilles d'exercices à imprimer ou des séquences sur un EIAH.

Les connaissances de création de séquences personnalisées contiennent d'une part les règles permettant de créer des feuilles d'exercices papier à partir des activités générées et d'autre part les règles permettant de créer pour les EIAH externes des séquences de travail valides.

La création de séquences de travail personnalisées correspond donc à une mise en forme des activités générées ou sélectionnées dans l'étape précédente pour fournir soit une feuille d'exercices papier à imprimer, soit une séance de travail sur un EIAH, soit les deux. Dans le cas des EIAH, les connaissances techniques des modèles OKEP/x sont nécessaires pour pouvoir créer des fichiers de configuration valides pour chacun des EIAH. En plus des séquences de travail destinées aux apprenants, le processus 
fournit à l'enseignant un bilan contenant le résumé des séquences proposées, et, dans le cas des activités papier, leur correction.

À la fin du processus de génération de séquences personnalisées, on obtient autant de feuilles d'exercices papier et/ou de configuration d'EIAH que l'on avait de profils d'apprenants. Les séquences créées sont toujours proposées à l'enseignant pour validation ou modification afin de lui laisser jusqu'au bout du processus sa place de pédagogue.

\section{Un logiciel de personnalisation des activités pédagogiques}

Dans cette section, nous présentons la mise en œuvre que nous avons faite du modèle PERSUA2 et de son processus d'exploitation, avant de présenter les évaluations menées sur nos propositions. Nous terminons la section en revenant sur les scénarios d'usage de la section 2 pour montrer en quoi nos propositions changent ces situations.

\subsection{Mise en ouvre et évaluations du modèle PERSUA2}

Le modèle PERSUA2 a été mis en œuvre dans Adapte, un logiciel intégré à l'environnement informatique EPROFILEA.

Le rôle de cet environnement est d'assister l'enseignant dans sa gestion de profils créés par l'enseignant lui-même ou issus d'EIAH (Jean-Daubias et al., 2009). Ces profils peuvent porter sur toutes les disciplines et tous les niveaux, scolaires ou universitaires. EPROFILEA comporte deux parties : la constitution de profils conformes à l'environnement et leur exploitation.

Le logiciel Adapte est l'une des exploitations possibles des profils : il permet de fournir à chaque apprenant des activités adaptées à son profil tout en respectant les choix pédagogiques de son enseignant. Ces activités peuvent être des activités papiercrayon proposées par le système ou des activités logicielles gérées par un autre EIAH (Lefevre et al., 2011a).

Comme Adapte respecte le modèle PERSUA2, son utilisation se fait en trois étapes : la définition d'une stratégie pédagogique par l'enseignant (cf. Figure 14), la définition du contexte d'utilisation par l'enseignant, et enfin la génération des séquences de travail personnalisées par le système (cf. Figure 15). Dans cette dernière étape, lorsque le système propose à l'enseignant les séquences de travail pour chaque apprenant, l'enseignant peut bien sûr les modifier si elles ne correspondent pas exactement à ses souhaits. 


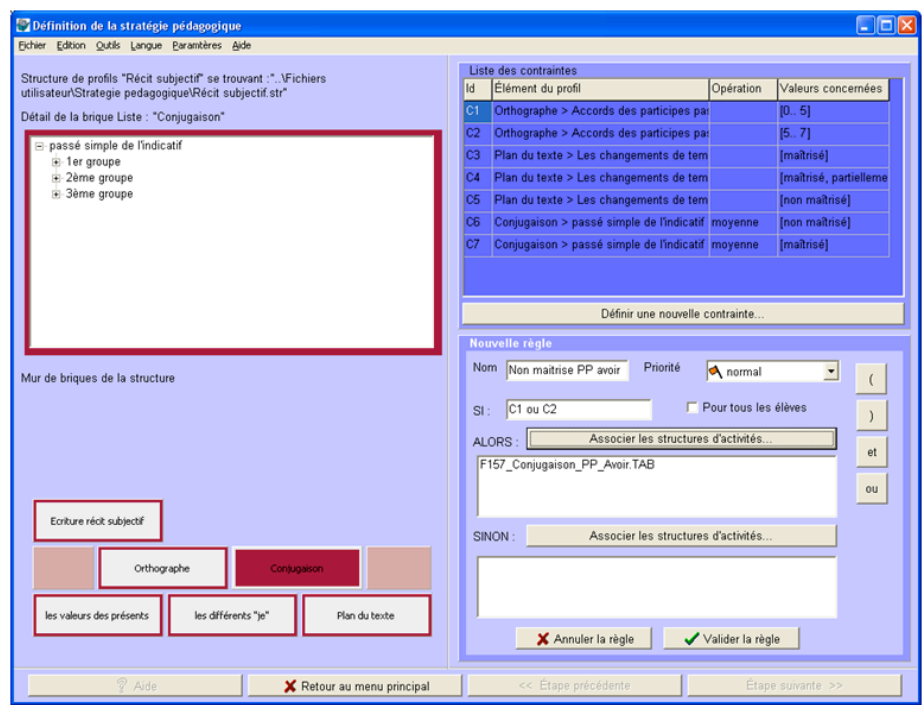

Figure 14 • Copie d'écran du logiciel Adapte : définition par un enseignant d'une stratégie pédagogique

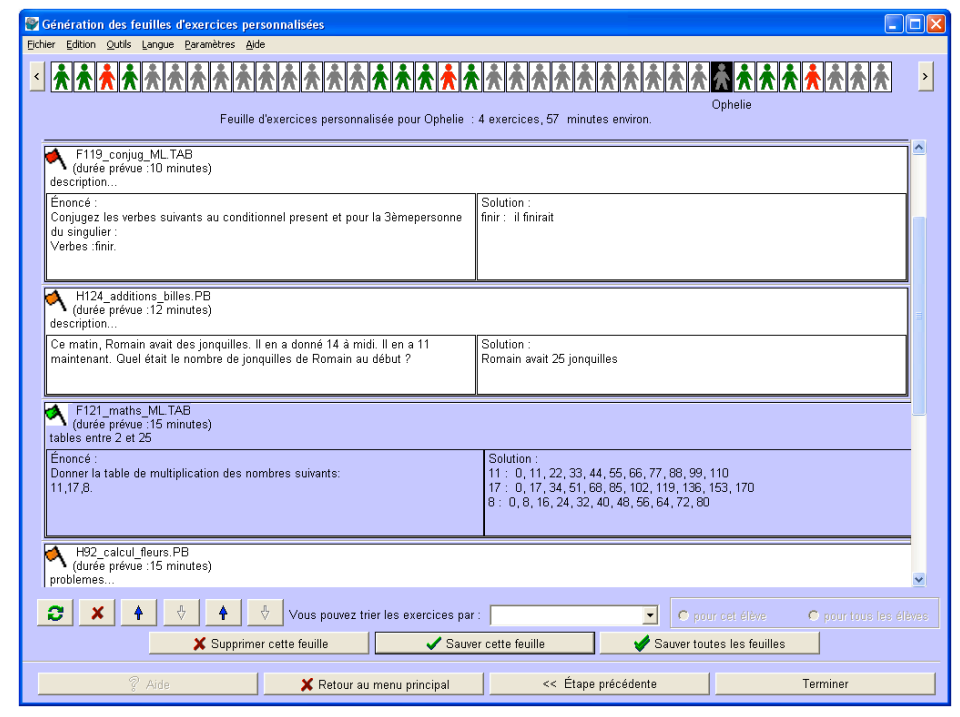

Figure 15 • Copie d'écran du logiciel Adapte : proposition par le logiciel d'activités personnalisées en fonction de la stratégie pédagogique de la Figure 14 
Cette mise en œuvre du modèle PERSUA2 et de son processus d'exploitation a d'une part montré leur faisabilité technique et nous a d'autre part permis de les évaluer par des mises en situation concrètes impliquant des enseignants.

Dans un premier temps, pendant une mise à l'essai de l'environnement EPROFILEA, nous avons évalué pendant une journée le modèle PERSUA2 permettant une personnalisation unifiée de l'apprentissage et le logiciel Adapte le mettant en œuvre. Cette évaluation s'est faite avec Bérengère, une enseignante de français au collège, que l'on peut qualifier de non experte puisque, d'une part, elle n'est jamais intervenue dans la conception des modèles ou des outils proposés dans le cadre de ce projet et, d'autre part, elle n'est pas une utilisatrice avancée de l'informatique.

Pour pouvoir évaluer le modèle PERSUA2, nous avons tout d'abord demandé à Bérengère de définir sur papier la façon dont elle utiliserait les profils d'apprenants qu'elle avait précédemment créés dans EPROFILEA, pour proposer des séquences de travail adaptées à chacun de ses élèves. Pendant deux heures, elle a ainsi défini des règles de personnalisation pouvant s'appliquer à des profils contenant 21 éléments. Ces éléments étaient définis pour neuf d'entre eux selon une échelle numérique (une note de 0 à 10 ) et pour les douze autres selon une échelle textuelle ordonnée (une liste énumérée de deux ou trois éléments selon les cas). Ces règles établissaient un lien entre la valeur d'un ou plusieurs des éléments du profil des élèves et des descriptifs d'exercices.

Une fois « son modèle » de personnalisation établi, nous avons demandé à Bérengère de le retranscrire dans le logiciel Adapte en créant la stratégie pédagogique correspondante. Pour cela, nous avons préalablement créé des structures d'activités correspondant à la description qu'elle avait faite des exercices. Cette étape préliminaire a été menée afin que l'évaluation de la partie principale d'Adapte (la création des règles d'affectation des activités aux apprenants), et donc du modèle PERSUA2, ne soit pas biaisée par des problèmes lors de la création des structures d'activités. La création de la stratégie pédagogique par Bérengère à partir de ses notes papier a duré une heure et a abouti à la création d'une stratégie pédagogique contenant 24 règles d'affectation des activités aux apprenants.

Le logiciel Adapte, et donc le modèle PERSUA2 sur lequel il s'appuie, ont permis à Bérengère de créer une stratégie pédagogique correspondant entièrement à ses notes papier. Néanmoins, nous avons dû lui expliquer comment utiliser les opérateurs « et, ou » afin de traduire certaines de ses règles de personnalisation. De plus, dans certains 
cas, elle aurait souhaité contraindre l'enchaînement des exercices proposés. Par exemple, pour des élèves ayant une difficulté donnée, leur faire faire l'exercice 1, puis s'ils ont réussi cet exercice, leur faire faire alors l'exercice 2, sinon l'exercice 3. Cette utilisation est une utilisation détournée du logiciel Adapte et le modèle PERSUA2 ne permet pas de créer ce type de règles. Toutefois, en modifiant l'énoncé des exercices, elle a pu décrire cette règle de la façon suivante « si un élève a telle difficulté alors lui faire faire les exercices 1, 2 et 3 ». Les contraintes sur l'enchaînement des exercices ont alors été intégrées au début du deuxième exercice par la phrase « $\mathrm{Si}$ vous avez réussi l'exercice précédent, passez à l'exercice 3, sinon faites uniquement cet exercice. ».

Une fois la stratégie pédagogique définie, nous avons lancé la génération des séquences personnalisées pour une de ses deux classes de $6^{\text {ème }}$. Pour cette classe de 23 élèves, la génération des séquences et leur enregistrement au format HTML a duré moins de 10 secondes. Afin de valider le processus associé au modèle PERSUA2 et sa mise en œuvre dans Adapte, nous avons étudié les 23 séquences d'activités générées et nous avons pu vérifier que chacune correspondait bien à la personnalisation que souhaitait mettre en place Bérengère.

Pour terminer cette évaluation, Bérengère a créé une seconde stratégie pédagogique sur une autre structure de profils d'apprenant, mais en utilisant cette fois directement le logiciel Adapte. Cette création a duré 25 minutes pour une stratégie contenant 13 règles.

Dans un second temps, nous avons effectué une évaluation similaire avec Hélène, une enseignante de classe spécialisée. Hélène devait dans sa pratique quotidienne proposer à la main des activités entièrement personnalisées pour chacun de ses cinq élèves en situation de handicap. L'évaluation s'est déroulée de la même manière : elle a permis à Hélène de définir sur papier, puis avec Adapte, une stratégie pédagogique adaptée à ses besoins. L'exécution de cette stratégie a permis la définition de séquences d'activités adaptées aux élèves d'Hélène.

Par ailleurs, nous avons mis Adapte à l'essai d'une part auprès d'une vingtaine de chercheurs dans le cadre de l'école d'été du CNRS consacrée à la personnalisation des EIAH, et d'autre part auprès d'une centaine d'étudiants de Master 1 Informatique suivant une option EIAH. Ces utilisateurs n'appartenant pas au public cible d'Adapte, ce test n'a pas de valeur de validation, mais il donne toutefois des indications sur l'utilité et l'utilisabilité de l'outil. Ces différents utilisateurs avaient pour consigne 
d'utiliser Adapte en adoptant le rôle d'un enseignant. Ils ont pu définir des stratégies pédagogiques, et les exécuter pour obtenir une séquence pédagogique entièrement personnalisée pour chaque apprenant.

\subsection{L'apport d'Adapte dans les scénarios}

Nous avons présenté au début de cet article trois scénarios montrant les enjeux de la personnalisation des activités pédagogiques dans les pratiques des enseignants, puis nous avons mis en évidence les freins actuels à leur mise en œuvre. Revenons à présent sur ces scénarios pour montrer en quoi le modèle PERSUA2 et l'implémentation qui a en été faite dans le logiciel Adapte pourraient s'intégrer aux trois scénarios, dans le but d'alléger la charge de travail de l'enseignant.

\subsubsection{Aide personnalisée à l'école primaire}

Dimitri, enseignant de CE2, encadre des séances de soutien pour les élèves en difficulté de son établissement. Pour préparer le contenu de ces séances, il souhaite s'appuyer sur toutes les informations dont ses collègues et lui disposent sur les connaissances des élèves (connaissances qu'ils ont des élèves, données issues des évaluations nationales et profils d'élèves créés par le logiciel AMBRE-add) pour proposer des séances de remédiation adaptées à chacun des élèves, soit sous forme papiercrayon, soit avec des logiciels d'apprentissage.

Avec l'environnement EProfiLEA, Dimitri et ses collègues peuvent récupérer la structure de profils correspondant aux évaluations nationales (c'est-à-dire le modèle des profils correspondants) et la fusionner avec celle correspondant au profil du logiciel AMBRE-add. Ils peuvent ainsi remplir cette unique structure de profils avec les données de tous les apprenants. Au final, les enseignants disposent d'un profil par élève contenant toutes les informations issues des évaluations nationales et du logiciel AMBRE-add. Chaque enseignant peut ensuite compléter la structure de profils pour ajouter les informations dont il a besoin, puis compléter le profil de ses élèves.

À partir de la structure de profils commune à tous les enseignants, chaque enseignant peut définir dans Adapte sa stratégie pédagogique pour le soutien, permettant de créer des activités uniquement sur papier, uniquement sur un ou plusieurs logiciels pédagogiques qu'il connait, ou combinant les deux supports.

L'enseignant encadrant la séance de soutien, ici Dimitri, peut ainsi, pour chaque élève présent, obtenir une séquence de travail adaptée aux buts pédagogiques de l'enseignant habituel de l'élève. Pour cela, Dimitri récupère la stratégie pédagogique 
de l'enseignant habituel de l'élève, définit le contexte d'utilisation propre à la séance de soutien (temps, matériel et logiciels disponibles) et demande la création d'une séance de travail adaptée au profil de l'élève. Dimitri peut faire cela pour les six élèves de sa séance.

Avec l'environnement EPROFILEA et le module Adapte, Dimitri et ses collègues peuvent mutualiser les informations dont ils disposent sur leurs élèves (informations issues des enseignements classiques ou des séances de soutien) et obtenir rapidement des séances de remédiation adaptées à chacun des élèves concernés. Après un travail de mise en place du dispositif, ils gagnent le temps de traitement de chaque profil puisqu'ils ne le font qu'une seule fois lors de la définition de la stratégie pédagogique. Le traitement des profils est ensuite fait à chaque fois par Adapte.

\subsubsection{Combiner remédiation et approfondissement}

Hélène, professeur d'anglais au collège, souhaite, avec chacune de ses classes, faire une séance d'une heure en salle informatique durant laquelle ses élèves travailleront une demi-heure sur des logiciels pédagogiques et une demi-heure avec elle en remédiation ou en approfondissement. Durant cette séance, elle souhaite que les logiciels qu'elle fait utiliser à ses classes aient des sessions adaptées aux besoins des élèves, ce qui n'est actuellement pas le cas.

Avec Eprofilea, Hélène peut définir une structure de profils, puis la compléter avec ses propres données afin de créer un profil par élève. Ensuite, dans Adapte, elle peut définir une stratégie pédagogique indiquant que les élèves ayant des difficultés de prononciation doivent travailler sur le logiciel Sephonics, tandis que les autres doivent apprendre du nouveau vocabulaire grâce aux logiciels Planet English et VocabOne. Pour chaque logiciel, elle peut paramétrer les activités que les élèves devront faire, en choisissant par exemple les thèmes pour le vocabulaire à étudier dans VocabOne. Pour obtenir des séquences de travail d'une demi-heure, elle définit son contexte d'utilisation. À partir du profil des élèves, Adapte peut ainsi lui proposer une séquence de travail adaptée à chaque élève sur un ou deux des logiciels pédagogiques.

En utilisant l'environnement EPROFILEA et plus particulièrement le module Adapte, Hélène peut créer des profils pour ses élèves afin d'obtenir des séquences de travail adaptées. L'avantage majeur de cette approche est qu'Hélène n'a pas à maîtriser trois outils, mais un seul pour personnaliser les trois logiciels pédagogiques. Ceci nécessite évidemment qu'une personne connaissant bien ces logiciels ait défini leurs 
modèles OKEP, ce qui est fait une seule fois et peut ensuite profiter à tous les enseignants utilisant Adapte.

\subsubsection{Plan licence à l'université}

Lucie est maître de conférences en informatique. Dans le cadre de ses enseignements, elle utilise un ENT contenant un profil pour chacun de ses étudiants ainsi qu'un ensemble de ressources pédagogiques.

Adapte peut utiliser les profils des étudiants de l'ENT pour proposer à chacun d'entre eux des ressources à consulter dans l'ENT. Pour cela, Lucie doit intégrer les profils de l'ENT à l'environnement EPROFILEA, puis définir dans Adapte sa stratégie pédagogique indiquant quelles ressources fournir en fonction du contenu ou de l'évolution du contenu du profil des étudiants.

Comme Dimitri, en utilisant Adapte, Lucie gagne le temps d'analyse de tous les profils des étudiants.

\section{Discussion}

Notre revue de l'existant montre qu'il n'y avait pas jusqu'à maintenant de tentative d'unification des approches permettant une personnalisation des activités d'apprentissage. En fonction des problématiques qu'ils abordent, les différents travaux existants ne traitent qu'une sous-partie de la question de la personnalisation de l'enseignement. Il n'existe donc aucun système, en dehors d'Adapte, permettant une personnalisation externalisée des activités papier et de plusieurs EIAH, en adaptant les séquences d'activités proposées aux apprenants à partir de leur profil, tout en laissant intervenir l'enseignant dans les choix de personnalisation. Toutefois, d'autres approches utilisent des règles pédagogiques d'adaptation à l'apprenant.

Ainsi, dans le projet LINGOT, des stratégies d'apprentissage sont proposées à l'enseignant pour chacun de ses apprenants. Pour cela, le système PépiStéréo (Vincent et al., 2005) attribue un stéréotype à chaque apprenant, chaque stéréotype ayant préalablement été associé à une stratégie d'apprentissage. Contrairement aux stratégies pédagogiques du modèle PERSUA2, qui vont permettre de créer pour chaque apprenant une séquence de travail personnalisée, les stratégies d'apprentissage du projet LINGOT contiennent une suite d'étapes permettant de faire travailler tous les apprenants associés à un stéréotype. Ces étapes contiennent des exemples d'exercices, ainsi qu'une liste d'EIAH pouvant être utilisés pour faire ce type d'exercices. Dans PépiStéréo, plusieurs apprenants pourront donc être associés à la 38 
même stratégie d'apprentissage et c'est l'enseignant qui devra construire la séquence de travail à partir des indications de la stratégie d'apprentissage. De plus, il existe un nombre limité de stratégies d'apprentissage et celles-ci sont spécifiques au domaine de l'algèbre élémentaire.

Si l'on revient sur les outils auteurs, plusieurs d'entre eux tels que REDEEM (Ainsworth, 2000), GTE (Van Marcke, 1998), Smart Trainer (Chen et al., 1998) et Eon (Murray, 2003a) permettent aux enseignants créant un logiciel pédagogique de paramétrer des modèles de stratégies pédagogiques. Certains de ces outils auteurs ont été définis en respectant le méta-modèle KBT-MM (Murray, 2003b). Ce méta-modèle est un guide permettant de proposer des outils auteurs qui permettent de construire des tuteurs intelligents. Dans les tuteurs créés, les connaissances relatives au contenu pédagogique sont séparées des stratégies pédagogiques. Mais les unes comme les autres sont incluses dans le tuteur créé. De la même manière, dans le domaine des hypermédias adaptatifs, des outils auteurs permettent de définir des hypermédias en séparant le contenu des stratégies d'adaptation. Par exemple, en utilisant de langage LAG (Cristea et Calvi, 2003), il est possible de définir des règles d'adaptation réutilisables pour des hypermédias adaptatifs construits selon le modèle LAOS (Cristea et De Mooij, 2003).

Le modèle PERSUA2 repose également sur une séparation du contenu pédagogique et des stratégies pédagogiques. Mais dans notre approche, un système exploitant le modèle PERSUA2, comme le logiciel Adapte, utilise des connaissances sur le contenu pédagogique de divers EIAH. Ces connaissances sont fournies par des experts grâce à l'approche GEPPETO qui permet d'acquérir ces connaissances et de les exploiter (Lefevre, 2010). En complément de ces connaissances, un système comme Adapte permet à l'enseignant de définir ses propres stratégies pédagogiques, et pas uniquement la stratégie pédagogique mise en place par le concepteur d'un EIAH qui aura utilisé un outil auteur respectant le méta-modèle KBT-MM ou le modèle LAOS. Contrairement aux stratégies définies selon ces modèles, celles définies selon le modèle PERSUA2 peuvent aussi porter sur plusieurs EIAH. Ainsi, le modèle PERSUA2 peut être utilisé dès lors que les EIAH sont configurables (via des fichiers de configuration ou des interfaces) et ce quel que soit leur type (tuteur intelligent, simulateur, micromonde...).

Ces deux approches reposent donc sur le même principe de séparation entre contenu et stratégies pédagogiques, mais le modèle PERSUA2 généralise les principes de KBT-MM ou de LAOS en permettant d'externaliser la définition des stratégies péda- 
gogiques non pas dans un outil auteur, mais dans un outil servant d'interface entre l'enseignant et les différents EIAH à configurer. Cette externalisation permet de personnaliser des logiciels existants, quel que soit leur provenance (logiciel conçu à partir d'outils auteurs ou directement), qui n'incluent pas de stratégie pédagogique à l'origine, mais également ceux qui ont des stratégies pédagogiques décrites dans des fichiers de configuration ou modifiables via une interface.

Le modèle de personnalisation PERSUA2 n'a à notre connaissance pas d'équivalent à l'heure actuelle. Il a été défini suite à des entretiens avec des enseignants pour identifier leurs pratiques en matière de personnalisation. Nous avions à la fin de la section 2 de cet article identifié un ensemble verrous à lever pour dépasser les limites des approches existantes en termes de personnalisation de l'apprentissage :

- Comment prendre en compte les individualités des apprenants? Comment diminuer la charge de travail d'un enseignant quand il traite les informations relatives aux apprenants?

- Comment prendre en compte les besoins et habitudes de travail des enseignants concernant le choix des activités à utiliser dans leur enseignement ? Et donc comment homogénéiser du point de vue de l'enseignant les outils de personnalisation des activités pédagogiques (générateurs d'exercices papier et interfaces de paramétrage des EIAH) ?

- Comment aider l'enseignant à affecter des activités en fonction des individualités des apprenants?

L'approche que nous avons présentée permet d'aborder ces verrous. Nous avons en effet montré que le logiciel Adapte permet à l'enseignant qui a explicité sa stratégie pédagogique d'être déchargé du traitement de chaque profil d'apprenant et de la génération d'activités adaptées. Cet enseignant n'a plus besoin d'utiliser plusieurs outils, Adapte lui permettant de générer des exercices papier, ainsi que des séquences sur des EIAH variés.

Le modèle PERSUA2 n'est pas exhaustif, mais il possède l'avantage d'être ouvert. Il a en effet été conçu afin d'être extensible et peut ainsi évoluer pour prendre en compte de nouvelles pratiques ou de nouveaux besoins éducatifs. Pour l'instant, le modèle PERSUA2 permet la personnalisation d'activités papier et de logiciels pédagogiques utilisés individuellement par les apprenants. Comme il est ouvert, il devrait permettre, à moindre coût, d'intégrer de nouveaux types d'EIAH, comme ceux proposant des situations d'apprentissage collaboratives ou collectives. 


\section{Conclusion}

La recherche présentée dans cet article consistait à définir un processus qui permette à la fois de personnaliser des séances de travail sur papier et des séances de travail sur des logiciels pédagogiques. Nous souhaitions que ce processus permette de prendre en compte les spécificités de chaque apprenant, en s'appuyant sur son profil, mais qu'il prenne également en compte les buts et les habitudes pédagogiques des enseignants. Enfin, nous souhaitions que ce processus puisse être mis en œuvre dans un système unique externe aux divers logiciels à personnaliser.

Pour répondre à cette problématique, nous avons proposé le modèle PERSUA2 qui permet de lier des contraintes sur profils à des contraintes sur activités. Ces liens, nommés règles d'affectation, sont ensuite hiérarchisés selon leur degré de priorité pour former une stratégie pédagogique qui sera associée à un ou plusieurs contextes d'utilisation. Nous avons mis en œuvre ce modèle dans Adapte, un logiciel intégré à l'environnement informatique EPROFILEA. Adapte permet de fournir des séquences de travail adaptées au profil de chaque élève et aux buts pédagogiques de l'enseignant.

Le modèle PERSUA2 est, à notre connaissance, le premier modèle permettant à un enseignant, quel que soit le domaine qu'il enseigne, de créer des séquences de travail propres à chaque apprenant, séquences pouvant être effectuées sur plusieurs supports, papier et/ou logiciels.

Les premières études menées sur le modèle PERSUA2 et sur sa mise en œuvre dans le logiciel Adapte ont montré l'utilité du modèle et la pertinence des personnalisations proposées par notre logiciel. Ces évaluations ont également mis en évidence la complexité de la tâche demandée aux enseignants lors de la formalisation de leurs règles de personnalisation. Pour cet aspect critique du processus de personnalisation, il apparaît donc essentiel d'assister l'enseignant avec une aide et des outils appropriés. Fournir une telle assistance est un processus complexe qui soulève plusieurs questions et oriente vers de nouvelles perspectives de recherche. Comment apporter une aide pertinente sans perturber l'enseignant dans sa tâche principale? Que nécessite une telle approche? De quelles connaissances le système d'assistance doit-il être doté pour que l'aide apportée soit à la fois personnalisée, pertinente dans le contexte de la tâche et cohérente avec les pratiques pédagogiques de l'enseignant ? Comment exploiter les interactions entre l'enseignant et le système pour acquérir de nouvelles connaissances et donc améliorer au fil des usages l'efficacité de l'assistance proposée ? Pour répondre à ces questions nous envisageons notamment de nous appuyer sur 
le paradigme du raisonnement à partir de l'expérience tracée (Cordier et al., 2010). Disposer d'un outil d'assistance flexible et répondant de façon simple aux besoins des utilisateurs est un atout majeur pour tout outil, en particulier parce qu'il accroît les chances que l'utilisateur s'habitue à l'environnement, le fasse sien et l'exploite à la hauteur des possibilités qu'il offre.

\section{BIBLIOGRAPHIE}

(Ainsworth, 2000)

AINSWORTH S. (2000). Redeem: Its authoring tools and human teaching. Workshop on Modelling Human Teaching Tactics, ITS'2000, Montréal, Canada.

(Bourdieu, 1980)

BOURDIEU P. (1980). Le sens pratique, Minuit.

(Brusilovsky, 2001)

BRUSILOVSKY P. (2001). Adaptive Hypermedia. User Modeling and User-Adapted Interaction, Vol. 11, p 87-110.

(Burton, 1982)

BURTON R. R. (1982). Diagnosing bugs in a simple procedural skill. Intelligent Tutoring Systems, London, Academic Press.

(Chen et al., 1998)

CHEN W., HAYASHI Y., JIN L., IKEDA M., MIZOGUCHI R. (1998). An Ontology-based Intelligent Authoring Tool. International Conference on Computers in Education (ICCE'98), p. 41-49.

(Clot et Faïta, 2000)

CLOT Y., FAÏTA D. (2000). Genres et styles en analyse du travail. Concetp et methods, Travailler, Vol. 4, p 7-42.

(Cordier et al., 2010)

CORDIER A., LEFEVRE M., JEAN-DAUBIAS S., GUIN N. (2010). , Concevoir des assistants intelligents pour des applications fortement orientées connaissances : problématiques, enjeux et étude de cas. $21^{\text {èmes }}$ Journées francophones d'Ingénierie des Connaissances (IC 2010), Nîmes, France.

(Cristea, 2005)

CRISTEA A. (2005). Authoring of Adaptative Hypermedia. Educational Technology \& Society, Vol. $8 \mathrm{n}^{\circ} 3$, p 6-8.

(Cristea et Calvi, 2003)

CRISTEA A., CALVI L. (2003). The three Layers of Adaptation Granularity. UM'03, Pittsburg, USA.

(Cristea et Carro, 2008)

CRISTEA A., CARRO R.-M. (2008). A3H: Fifth International Workshop on Authoring of Adaptive and Adaptable Hypermedia. Fifth International Workshop on Authoring of Adaptive and Adaptable Hypermedia (UM2008), Hannover, Germany, p. 1-84.

\section{2}


(Cristea et De Mooij, 2003)

CRISTEA A., MOOIJ A. (2003). LAOS: Layered WWW AHS Authoring Model and their corresponding Algebraic Operators. The Twelfth International World Wide Web Conference (WWW03), Alternate Track on Education, Budapest, Hungary.

(David et al., 1996)

DAVID J.-P., COGNE A., DUTEL A. (1996). Hypermedia exercises prototyping and modelising. Computer Aided Learning and Instruction in Science and Engineering. Heidelberg, S. B., p. 252-260.

(Duclosson et al., 2005)

DUCLOSSON N., JEAN-DAUBIAS S., RIOT S. (2005). AMBRE-enseignant : un module partenaire de l'enseignant pour créer des problèmes. Environnements Informatiques pour l'Apprentissage Humain (EIAH'2005), Montpellier, France, p. 353-358.

(Eyssautier-Bavay, 2008)

EYSSAUTIER-BAVAY C. (2008). Modèles, langage et outils pour la réutilisation de profils d'apprenants. Thèse de doctorat en Informatique, Université Joseph Fourier.

(Ginon et Jean-Daubias, 2010)

GINON B., JEAN-DAUBIAS S. (2010). Étude du concept de profils évolutifs dans le cadre du projet PERLEA. Rapport de recherche LIRIS UMR 5205, RR-LIRIS-2010-007.

(Girard et al., 2007)

GIRARD S., JOHNSON H. (2007). DividingQuest: opening the learner model to teachers. Environnements Informatiques pour l'Apprentissage Humain (EIAH'2007), Lausanne, Suisse, p. 329-334.

(Guin et al., 2011)

GUIN N., LEFEVRE M., JEAN-DAUBIAS S. (2011). Personnalisation de l'apprentissage dans l'EIAH Ambre-add. Atelier "Personnalisation de l'apprentissage : quelles approches pour quels besoins ?", EIAH 2011, Mons, Belgique.

(J'ADE, 2007)

J'ADE (2007). Guide d'installation et d'utilisation, Ministère Français de l'éducation nationale, de l'enseignement supérieur et de la recherche.

(Jean-Daubias, 2011)

JEAN-DAUBIAS S. (2011). Ingénierie des profils d'apprenants. Habilitation à diriger des recherches, Université Claude Bernard Lyon 1, France.

(Jean-Daubias et al., 2009)

JEAN-DAUBIAS S., EYSSAUTIER-BAVAY C., LEFEVRE M. (2009). Modèles et outils pour rendre possible la réutilisation informatique de profils d'apprenants hétérogènes. Revue Sciences et Technologies de l'Information de de la Communication pour l'Education et la Formation (STICEF), Vol. 16.

(Jean-Daubias et Ginon, 2010)

JEAN-DAUBIAS S., GINON B. (2010). Des profils d'apprenants évolutifs. TICE 2010, Nancy, France.

(Lefevre, 2009)

LEFEVRE M. (2009). Processus unifié pour la personnalisation des activités pédagogiques : méta-modèle, modèles et outils. Thèse de doctorat, Université Claude Bernard Lyon 1, France. 
(Lefevre, 2010)

LEFEVRE M. (2010). GEPPETO : une approche générique permettant d'adapter les activités des apprenants aux intentions pédagogiques de chaque enseignant. 3ème Rencontres Jeunes Chercheurs en Environnements Informatiques pour l'Apprentissage Humain (RJC EIAH 2010), Lyon, France, p. 35-40.

(Lefevre et al., 2009)

LEFEVRE M., MILLE A., JEAN-DAUBIAS S., GUIN N. (2009). A Meta-Model to Acquire Relevant Knowledge for Interactive Learning Environments Personalization. Adaptive 2009, Athènes, Grèce.

(Lefevre et al., 2011a)

LEFEVRE M., JEAN-DAUBIAS S., GUIN N. (2011). Adapte, un logiciel pour aider l'enseignant à proposer des activités personnalisées à chacun de ses apprenants. Atelier Personnalisation de l'apprentissage, EIAH', Mons, Belgique. Disponible sur internet: http://liris.cnrs.fr/marie.lefevre/public_html/doku.php?id=ateliereiah2011 (consulté le 21 juillet 2011).

(Lefevre et al., 2011b)

LEFEVRE M., JEAN-DAUBIAS S., GUIN N. (2011). Actes de l'Atelier "Personnalisation de l'apprentissage : quelles approches pour quels besoins ?", EIAH 2011, Mons, Belgique, disponibles sur le site http://liris.cnrs.fr/marie.lefevre/public_html/doku.php?id=ateliereiah2011.

(Leroux, 2002)

LEROUX P. (2002). Machines partenaires des apprenants et des enseignants - Étude dans le cadre d'environnements supports de projets pédagogiques. Habilitation à Diriger des Recherches en Informatique, Université du Maine.

(Marty et Mille, 2009)

MARTY J-C., MILLE A. (2009). Analyse de traces et personnalisation des environnements informatiques pour l'apprentissage humain. Traité IC2 : Informatique et systèmes d'information, Hermès Sciences.

(Melis et al., 2001)

MELIS E., ANDRÉS E., BÜDENBENDER J., FRISCHAUF A., GOGUADZE G., LIBBRECHT P., POLLET M., ULLRICH C. (2001). ActiveMath: A Generic and Adaptive Web-Based Learning Environment. International Journal of Artificial Intelligence in Education (IJAIED), Vol. 12, p. 385-407.

(Mitrovic, 1998)

MITROVIC A. (1998). A Knowledge-Based Teaching System for SQL. Association for the Advancement of Computing in Education (AACE).

(Murray, 2003a)

MURRAY T. (2003). Eon: Authoring Tools for Content, Instructional Strategy, Student Model, and Interface Design. Authoring Tools for Advanced Technology Learning Environments, Chap. 11, Murray, T., Blessing, S. \& Ainsworth, S. (Eds.), Kluwer Academic Publisher.

(Murray, 2003b)

MURRAY T. (2003). Principles for Pedagogy-oriented Knowledge Based Tutor Authoring Systems: Lessons Learned and a Design Meta-Model. Authoring Tools for Advanced Technology Learning Environments, Chap. 15, Murray, T., Blessing, S. \& Ainsworth, S. (Eds.), Kluwer Academic Publisher. 
(Pernin et al., 2004)

PERNIN J.-P., LEJEUNE A. (2004). Dispositifs d'apprentissage Instrumentés par les Technologies : vers une ingénierie centrée sur les scénarios. Technologies de l'Information et de la Connaissance dans l'Enseignement Supérieur et l'Industrie (TICE'2004), Compiègne, France.

(Perrenoud, 2000)

PERRENOUD P. (2000). La Formation des enseignants entre théorie et pratique. Savoir et Formation, L'harmattan.

(Rich, 1983)

RICH E. (1983). Users are individuals: individualizing user models. International Journal of Man-Machine Studies, Vol. 18 n³, p 199-214.

(Sørmo et al., 2002)

SØRMO F., AAMODT A. (2002). Knowledge communication and CBR. 6th European Conference on Case-Based Reasoning (ECCBR 2002), p. 47-59.

(Van Joolingen et al., 2003)

VAN JOOLINGEN W.-R., DE JONG T. (2003). Simquest: Authoring educational simulations. Authoring tools for advanced technology educational software: Toward cost-effective production of adaptive, interactive, and intelligent educational software, Murray, T., Blessing, S. \& Ainsworth, S. (Eds.), Kluwer Academic Publisher, p. 1-31.

(Van Marcke, 1998)

VAN MARCKE K. (1998). GTE: An epistemological approach to instructional modeling. Instructional Science, Vol. 26 n³-4, p. 147-191.

(VanLehn et al., 2005)

VANLEHN K., LYNCH C., SCHULZE K., SHAPIRO J. A., SHELBY R., TAYLOR L., TREACY D., WEINSTEIN A., WINTERSGILL M. (2005). The Andes Physics Tutoring System: Lessons Learned. International Journal of Artificial Intelligence in Education, Vol. 15, p. 147-204.

(Vincent et al., 2005)

VINCENT C., DELOZANNE E., GRUGEON B., GELIS J.-M., ROGALSKI J., COULANGE L. (2005). Des erreurs aux stéréotypes : Des modèles cognitifs de différents niveaux dans le projet Pépite. Environnements Informatiques pour l'Apprentissage Humain (EIAH'2005), Montpellier, France, p. 297-308.

(Wenger, 1987)

WENGER E. (1987). Artificial Intelligence and Tutoring Systems. Los Altos, CA, Morgan Kaufmann.

\section{NETOGRAPHIE}

(iClass, 2004)

ICLASS (2004). Intelligent Distributed Cognitive-based Open Learning System for Schools,

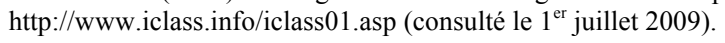

(Planet English, 2006)

PLANET ENGLISH (2006). http://www.anglaisfacile.com/free/lejeu/index.php (consulté le 21 juillet 2011). 
(Sephonics, 2008)

SEPHONICS (2008). Sephonics - Learn The IPA Alphabet http://www.wartoft.nu/software/sephonics/ (consulté le 21 juillet 2011).

(VocabOne, 2007)

VOCABONE (2007). VocabOne 02. http://www.zeniko.ch/static/vocabone.html (consulté le 21 juillet 2011).

\section{9. À propos des auteurs}

Marie Lefevre est en post-doctorat au LIRIS au sein de l'équipe SILEX (Supporting Interaction and Learning by Experience). Ses recherches portent sur la personnalisation de l'apprentissage, et notamment sur la proposition de modèles et d'outils génériques permettant à chaque enseignant d'obtenir, pour chacun de ses élèves, des activités pédagogiques adaptées d'une part à ses intentions pédagogiques et d'autre part au profil de l'élève. Elle s'intéresse également à l'étude des interactions utilisateur pour la construction d'outils d'assistance dynamiques et adaptables, notamment au sein de wikis sémantiques distribués.

Adresse : Université de Lyon, CNRS - Université Lyon 1, LIRIS, UMR5205, F69622, France

Courriel : Marie.Lefevre@liris.cnrs.fr

Toile : http://liris.cnrs.fr/marie.lefevre/

Nathalie Guin est maître de conférences en informatique à l'Université de Lyon. Elle est rattachée au LIRIS (Laboratoire d'InfoRmatique en Images et Systèmes d'information, UMR 5205) au sein de l'équipe SILEX (Supporting Interaction and Learning by Experience). Ses recherches portent sur les systèmes à base de connaissances pour les EIAH. Ses thèmes d'intérêt actuels sont liés à la personnalisation des EIAH : interprétation des traces d'activités, profils d'apprenants, génération d'activités adaptées. L'ensemble des ces thématiques de recherches sont mises en œuvre au sein du projet AMBRE.

Adresse : Université de Lyon, Lyon, F-69003, France ; Université Lyon 1, Lyon, F-69003, France ; LIRIS - UMR5205, Villeurbanne, F-69622, France

Courriel : Nathalie.Guin@liris.univ-lyon1.fr 
Toile : http://liris.cnrs.fr/nathalie.guin

Stéphanie Jean-Daubias est maître de conférences en informatique à l'Université Claude Bernard Lyon 1. Elle est rattachée au LIRIS (Laboratoire d'InfoRmatique en Images et Systèmes d'information, UMR 5205) au sein de l'équipe SILEX (Supporting Interaction and Learning by Experience). Ses recherches portent sur l'ingénierie des profils d'apprenants et la personnalisation des apprentissages. Elles sont mises en œuvre, principalement au sein des projets PERLEA et AMBRE, sous forme d'outils d'assistance destinés aux enseignants.

Adresse : Université de Lyon, CNRS - Université Lyon 1, LIRIS, UMR5205, F69622, France

Courriel : Stephanie.Jean-Daubias@liris.univ-lyon1.fr

Toile : http://liris.cnrs.fr/stephanie.jean-daubias/

\footnotetext{
${ }^{1}$ L'identifiant des types d'activités (par exemple P B17) est créé de la façon suivante : une première lettre indiquant s'il s'agit d'activités papier (P) ou logicielles (S) ; une seconde lettre représentant le générateur ou l'EIAH concerné (par exemple B pour des activités portant sur des illustrations); un numéro incrémental qui permet de rendre l'identifiant unique ; éventuellement des compléments textuels saisis librement par le créateur du type d'activités.

${ }^{2}$ Le terme OKEP/x signifie « Operational Knowledge Enabling Personalization of the ILE $\mathrm{x}$ » où ILE signifie « Interactive Learning Environments ».

${ }^{3}$ Le terme AKEPI signifie « Acquisition of Knowledge Enabling Personalization of ILEs » où ILE signifie « Interactive Learning Environments ».
} 